\title{
On the Computation of the Survival Probability of Brownian Motion with Drift in a Closed Time Interval When the Absorbing Boundary Is a Step Function
}

\author{
Tristan Guillaume \\ Université de Cergy-Pontoise, Laboratoire Thema, 33 boulevard du port, 95011 Cergy-Pontoise Cedex, France \\ Correspondence should be addressed to Tristan Guillaume; tristan.guillaume@u-cergy.fr
}

Received 7 April 2015; Accepted 13 May 2015

Academic Editor: Dejian Lai

Copyright (C) 2015 Tristan Guillaume. This is an open access article distributed under the Creative Commons Attribution License, which permits unrestricted use, distribution, and reproduction in any medium, provided the original work is properly cited.

This paper provides explicit formulae for the probability that an arithmetic or a geometric Brownian motion will not cross an absorbing boundary defined as a step function during a finite time interval. Various combinations of downward and upward steps are handled. Numerical computation of the survival probability is done quasi-instantaneously and with utmost precision. The sensitivity of the survival probability to the number and the ordering of the steps in the boundary is analyzed.

\section{Introduction}

The question of the first passage time of a diffusion process to an absorbing boundary is of central importance in many mathematical sciences. As mentioned in Wang and Pötzelberger [1], it arises in biology, economics, engineering reliability, epidemiology, finance, genetics, seismology, and sequential statistical analysis. Typically, one needs to compute the probability that some random dynamics modelled as a diffusion process will remain under or above some critical threshold over a given time interval. This is often referred to as a survival probability. Some papers focus on specific forms of the boundary, for example, a square root one (Breiman [2] and Sato [3]) or a curved one (Groeneboom [4] and Daniels [5]). Other contributions focus on a specific diffusion process, mostly Brownian motion (Park and Schuurmann [6], Jennen and Lerche [7], Salminen [8], Scheike [9], Novikov et al. [10], and Fu and $\mathrm{Wu}$ [11], 2010) or Ornstein-Uhlenbeck process (Alili et al. [12] and Lo and Hui [13]). Alternatively, some papers provide results for general classes of diffusion processes or boundaries (Durbin [14], Pötzelberger and Wang [15], Wang and Pötzelberger [1], and Downes and Borovkov [16]).

The contributions mentioned in this short noncomprehensive survey either focus on numerical algorithms usually involving recursive multidimensional quadrature or they seek to obtain approximate solutions by substituting the initial boundary with another one for which computations are easier and then deriving a bound for the error entailed by using the approximating boundary. This is because no closed form solution is known except in very few special cases when the boundary is linear and the underlying process is a Brownian motion. The main contribution of this paper is to provide a closed form solution to the problem of the computation of the survival probability of an arithmetic or geometric Brownian when the boundary is a step function of time. In general, this problem is numerically approximated by Monte Carlo simulation. The reason for this is twofold: from an analytical standpoint, the calculations involved are cumbersome and relatively complicated; from a numerical standpoint, the dimension of the integrals involved increases linearly with the number of steps in the boundary. In this paper, explicit formulae are provided up to five steps. An extension to higher dimensions is analytically straightforward. These formulae can be numerically computed quasi instantaneously with utmost precision in contrast to the slow and inaccurate approximations yielded by a Monte Carlo simulation. Nonmonotonic sequences of upper and lower steps are handled. The sensitivity of the survival probability with regard to the number and the ordering of the steps 
in the boundary is examined essentially as a function of the volatility of the underlying process. The problem of the location of the maximum or the minimum of Brownian motion with drift over several time intervals is also tackled. Although the purpose of this paper is not to study a specific engineering problem, one can mention that an immediate application of the results derived in this paper is the pricing and the hedging of many kinds of popular financial contracts known as barrier options and lookback options.

Section 2 provides a formula for the survival probability of an arithmetic Brownian motion when the absorbing boundary includes three steps. Section 3 is dedicated to numerical experiments whose purpose is to gain insight into the sensitivity of the survival probability to the number and the ordering of the steps. Section 4 extends the results of Section 2 to higher dimensions of the absorbing boundary. Section 5 provides a generalization to two-sided absorbing boundaries.

\section{Formula for the Survival Probability of an Arithmetic Brownian Motion through a Sequence of Three Absorbing Steps}

Let $\mu$ be a real constant and let $\sigma$ be a positive real constant. Let $\{B(t), t \geq 0\}$ be a standard Brownian motion. Under a given probability measure $\mathbb{P}$, let $X(t)$ be the process driven by

$$
d X(t)=\mu d t+\sigma d B(t) .
$$

A finite time interval $[0, T]$ is considered and divided into $n$ subintervals $\left[t_{0}=0, t_{1}\right] \cdots\left[t_{n-1}, t_{n}=T\right], n \in \mathbb{N}$. Let us define a piecewise constant boundary made up of $n$ horizontal line segments matching $n$ time subintervals, in other words, a step function. These line segments are called the steps of the boundary. They take on constant values $H_{i}$ in each time subinterval $\left[t_{i-1}, t_{i}\right], i \in\{1, \ldots, n\}$. The boundary may or may not be monotonically increasing or decreasing in time. When $H_{i}>X(0)$, the step is said to be upward, and when $H_{i}<X(0)$, it is said to be downward. When all the steps of the boundary are upward, the boundary itself is said to be upward and may also be called an upper boundary, while a downward boundary (also referred to as a lower boundary) is one whose steps are all downward.

The process $X$ is said to have survived at maturity $t_{n}$ when none of the steps has been hit at any moment in continuous time from $t_{0}$ to $t_{n}$.

The tractability of the calculation of the survival probability depends on the number of steps in the boundary. Indeed, each additional step increases the dimension of the integration problem under consideration. We first come up with a general closed form solution for boundaries made up of three steps. Higher dimensions are considered in Section 3.

Let $P_{[\ldots]}\left(h_{1}, h_{2}, h_{3}, k_{1}, k_{2}, k_{3}, t_{1}, t_{2}, t_{3}\right)$ be defined as one of the six following cumulative distribution functions, where $t_{3} \geq t_{2} \geq t_{1} \geq t_{0}=0$ and $k_{1}, k_{2}, k_{3}$ are real constants:

$$
\begin{aligned}
& P_{[M M M]}\left(h_{1}, h_{2}, h_{3}, k_{1}, k_{2}, k_{3}, t_{1}, t_{2}, t_{3}\right) \triangleq \mathbb{P}\left(\sup _{0 \leq t \leq t_{1}} X(t)\right. \\
& \quad \leq h_{1}, X\left(t_{1}\right) \leq k_{1}, \sup _{t_{1} \leq t \leq t_{2}} X(t) \leq h_{2}, X\left(t_{2}\right) \leq k_{2}, \sup _{t_{2} \leq t \leq t_{3}} X(t) \\
& \left.\quad \leq h_{3}, X\left(t_{3}\right) \leq k_{3}\right)
\end{aligned}
$$

$h_{1} \in \mathbb{R}_{+},\left(h_{2}, h_{3}, k_{1}, k_{2}, k_{3}\right) \in \mathbb{R}^{5}, k_{1} \leq h_{1}, k_{2} \leq h_{2}, k_{3} \leq h_{3}$,

$$
\begin{aligned}
& P_{[M M m]}\left(h_{1}, h_{2}, h_{3}, k_{1}, k_{2}, k_{3}, t_{1}, t_{2}, t_{3}\right) \triangleq \mathbb{P}\left(\sup _{0 \leq t \leq t_{1}} X(t)\right. \\
& \quad \leq h_{1}, X\left(t_{1}\right) \leq k_{1}, \sup _{t_{1} \leq t \leq t_{2}} X(t) \leq h_{2}, X\left(t_{2}\right) \leq k_{2}, \inf _{t_{2} \leq t \leq t_{3}} X(t) \\
& \left.\quad \geq h_{3}, X\left(t_{3}\right) \geq k_{3}\right)
\end{aligned}
$$

$h_{1} \in \mathbb{R}_{+},\left(h_{2}, h_{3}, k_{1}, k_{2}, k_{3}\right) \in \mathbb{R}^{5}, k_{1} \leq h_{1}, k_{2} \leq h_{2}, k_{3} \geq h_{3}$,

$$
\begin{aligned}
& P_{[\mathrm{Mmm}]}\left(h_{1}, h_{2}, h_{3}, k_{1}, k_{2}, k_{3}, t_{1}, t_{2}, t_{3}\right) \triangleq \mathbb{P}\left(\sup _{0 \leq t \leq t_{1}} X(t)\right. \\
& \quad \leq h_{1}, X\left(t_{1}\right) \leq k_{1}, \inf _{t_{1} \leq t \leq t_{2}} X(t) \geq h_{2}, X\left(t_{2}\right) \geq k_{2}, \inf _{t_{2} \leq t \leq t_{3}} X(t) \\
& \left.\quad \geq h_{3}, X\left(t_{3}\right) \geq k_{3}\right)
\end{aligned}
$$$$
h_{1} \in \mathbb{R}_{+},\left(h_{2}, h_{3}, k_{1}, k_{2}, k_{3}\right) \in \mathbb{R}^{5}, k_{1} \leq h_{1}, k_{2} \geq h_{2}, k_{3} \geq h_{3}
$$$$
P_{[m m m]}\left(h_{1}, h_{2}, h_{3}, k_{1}, k_{2}, k_{3}, t_{1}, t_{2}, t_{3}\right) \triangleq \mathbb{P}\left(\inf _{0 \leq t \leq t_{1}} X(t)\right.
$$$$
\geq h_{1}, X\left(t_{1}\right) \geq k_{1}, \inf _{t_{1} \leq t \leq t_{2}} X(t) \geq h_{2}, X\left(t_{2}\right) \geq k_{2}, \inf _{t_{2} \leq t \leq t_{3}} X(t)
$$$$
\left.\geq h_{3}, X\left(t_{3}\right) \geq k_{3}\right)
$$$$
h_{1} \in \mathbb{R}_{-},\left(h_{2}, h_{3}, k_{1}, k_{2}, k_{3}\right) \in \mathbb{R}^{5}, k_{1} \geq h_{1}, k_{2} \geq h_{2}, k_{3} \geq h_{3} \text {, }
$$$$
P_{[m m M]}\left(h_{1}, h_{2}, h_{3}, k_{1}, k_{2}, k_{3}, t_{1}, t_{2}, t_{3}\right) \triangleq \mathbb{P}\left(\inf _{0 \leq t \leq t_{1}} X(t)\right.
$$$$
\geq h_{1}, X\left(t_{1}\right) \geq k_{1}, \inf _{t_{1} \leq t \leq t_{2}} X(t) \geq h_{2}, X\left(t_{2}\right) \geq k_{2}, \sup _{t_{2} \leq t \leq t_{3}} X(t)
$$$$
\left.\leq h_{3}, X\left(t_{3}\right) \leq k_{3}\right)
$$

$$
h_{1} \in \mathbb{R}_{-},\left(h_{2}, h_{3}, k_{1}, k_{2}, k_{3}\right) \in \mathbb{R}^{5}, k_{1} \geq h_{1}, k_{2} \geq h_{2}, k_{3} \leq h_{3} \text {, }
$$$$
P_{[m M M]}\left(h_{1}, h_{2}, h_{3}, k_{1}, k_{2}, k_{3}, t_{1}, t_{2}, t_{3}\right) \triangleq \mathbb{P}\left(\inf _{0 \leq t \leq t_{1}} X(t)\right.
$$$$
\geq h_{1}, X\left(t_{1}\right) \geq k_{1}, \sup _{t_{1} \leq t \leq t_{2}} X(t) \leq h_{2}, X\left(t_{2}\right) \leq k_{2}, \sup _{t_{2} \leq t \leq t_{3}} X(t)
$$$$
\left.\leq h_{3}, X\left(t_{3}\right) \leq k_{3}\right)
$$

$h_{1} \in \mathbb{R}_{-},\left(h_{2}, h_{3}, k_{1}, k_{2}, k_{3}\right) \in \mathbb{R}^{5}, k_{1} \geq h_{1}, k_{2} \leq h_{2}, k_{3} \leq h_{3}$.

Then, we have the following. 


\section{Proposition 1.}

$$
\begin{aligned}
& P_{[\cdots]}\left(h_{1}, h_{2}, h_{3}, k_{1}, k_{2}, k_{3}, t_{1}, t_{2}, t_{3}\right) \\
& =N_{3}\left[\frac{\left(I_{1}+I_{2}+I_{3}\right)\left(\left(I_{1}+I_{2}\right)\left(k_{1} \wedge h_{2}\right)+I_{3} k_{1}-\mu t_{1}\right)-\left(I_{4}+I_{5}+I_{6}\right)\left(\left(I_{4}+I_{5}\right)\left(k_{1} \vee h_{2}\right)+I_{6} k_{1}-\mu t_{1}\right)}{\sigma \sqrt{t_{1}}},\right. \\
& \frac{\left(I_{1}+I_{2}+I_{6}\right)\left(\left(I_{1}+I_{6}\right)\left(k_{2} \wedge h_{3}\right)+I_{2} k_{2}-\mu t_{2}\right)-\left(I_{3}+I_{4}+I_{5}\right)\left(\left(I_{3}+I_{4}\right)\left(k_{2} \vee h_{3}\right)+I_{5} k_{2}-\mu t_{2}\right)}{\sigma \sqrt{t_{2}}}, \\
& \frac{\left(I_{1}+I_{5}+I_{6}-I_{2}-I_{3}-I_{4}\right)\left(k_{3}-\mu t_{3}\right)}{\sigma \sqrt{t_{3}}} ;\left(I_{1}+I_{2}-I_{3}+I_{4}+I_{5}-I_{6}\right) \sqrt{\frac{t_{1}}{t_{2}}},\left(I_{1}-I_{2}-I_{3}+I_{4}-I_{5}-I_{6}\right) \sqrt{\frac{t_{1}}{t_{3}}}, \\
& \left.\left(I_{1}-I_{2}+I_{3}+I_{4}-I_{5}+I_{6}\right) \sqrt{\frac{t_{2}}{t_{3}}}\right]-\left(I_{2}+I_{3}+I_{5}+I_{6}\right) \\
& \cdot N_{3}\left[\frac{\left(I_{2}+I_{3}\right)\left(I_{2}\left(k_{1} \wedge h_{2}\right)+I_{3} h_{2}-\mu t_{1}\right)-\left(I_{5}+I_{6}\right)\left(I_{5}\left(k_{1} \vee h_{2}\right)+I_{6} h_{2}-\mu t_{1}\right)}{\sigma \sqrt{t_{1}}},\right. \\
& \frac{\left(I_{2}+I_{6}\right)\left(I_{2} h_{3}+I_{6}\left(k_{2} \wedge h_{3}\right)-\mu t_{2}\right)-\left(I_{3}+I_{5}\right)\left(I_{3}\left(k_{2} \vee h_{3}\right)+I_{5} h_{3}-\mu t_{2}\right)}{\sigma \sqrt{t_{2}}}, \frac{\left(I_{5}+I_{6}-I_{2}-I_{3}\right)\left(k_{3}-\mu t_{3}\right)}{\sigma \sqrt{t_{3}}} ; \\
& \left.\left(I_{1}+I_{2}-I_{3}+I_{4}+I_{5}-I_{6}\right) \sqrt{\frac{t_{1}}{t_{2}}},\left(I_{1}-I_{2}-I_{3}+I_{4}-I_{5}-I_{6}\right) \sqrt{\frac{t_{1}}{t_{3}}},\left(I_{1}-I_{2}+I_{3}+I_{4}-I_{5}+I_{6}\right) \sqrt{\frac{t_{2}}{t_{3}}}\right]-\exp \left(\frac{2 \mu h_{1}}{\sigma^{2}}\right) \\
& \cdot N_{3}\left[\frac{\left(I_{1}+I_{2}+I_{3}\right)\left(\left(I_{1}+I_{2}\right)\left(k_{1} \wedge h_{2}\right)+I_{3} k_{1}-2 h_{1}-\mu t_{1}\right)-\left(I_{4}+I_{5}+I_{6}\right)\left(\left(I_{4}+I_{5}\right)\left(k_{1} \vee h_{2}\right)+I_{6} k_{1}-2 h_{1}-\mu t_{1}\right)}{\sigma \sqrt{t_{1}}},\right. \\
& \frac{\left(I_{1}+I_{2}+I_{6}\right)\left(\left(I_{1}+I_{6}\right)\left(k_{2} \wedge h_{3}\right)+I_{2} k_{2}-2 h_{1}-\mu t_{2}\right)-\left(I_{3}+I_{4}+I_{5}\right)\left(\left(I_{3}+I_{4}\right)\left(k_{2} \vee h_{3}\right)+I_{5} k_{2}-2 h_{1}-\mu t_{2}\right)}{\sigma \sqrt{t_{2}}}, \\
& \frac{\left(I_{1}+I_{5}+I_{6}-I_{2}-I_{3}-I_{4}\right)\left(k_{3}-2 h_{1}-\mu t_{3}\right)}{\sigma \sqrt{t_{3}}} ;\left(I_{1}+I_{2}-I_{3}+I_{4}+I_{5}-I_{6}\right) \sqrt{\frac{t_{1}}{t_{2}}},\left(I_{1}-I_{2}-I_{3}+I_{4}-I_{5}-I_{6}\right) \sqrt{\frac{t_{1}}{t_{3}}}, \\
& \left.\left(I_{1}-I_{2}+I_{3}+I_{4}-I_{5}+I_{6}\right) \sqrt{\frac{t_{2}}{t_{3}}}\right]+\left(I_{2}+I_{3}+I_{5}+I_{6}\right) \exp \left(\frac{2 \mu h_{1}}{\sigma^{2}}\right) \\
& \cdot N_{3}\left[\frac{\left(I_{2}+I_{3}\right)\left(I_{2}\left(k_{1} \wedge h_{2}\right)+I_{3} h_{2}-2 h_{1}-\mu t_{1}\right)-\left(I_{5}+I_{6}\right)\left(I_{5}\left(k_{1} \vee h_{2}\right)+I_{6} h_{2}-2 h_{1}-\mu t_{1}\right)}{\sigma \sqrt{t_{1}}},\right. \\
& \frac{\left(I_{2}+I_{6}\right)\left(I_{2} h_{3}+I_{6}\left(k_{2} \wedge h_{3}\right)-2 h_{1}-\mu t_{2}\right)-\left(I_{3}+I_{5}\right)\left(I_{3}\left(k_{2} \vee h_{3}\right)+I_{5} h_{3}-2 h_{1}-\mu t_{2}\right)}{\sigma \sqrt{t_{2}}}, \\
& \frac{\left(I_{5}+I_{6}-I_{2}-I_{3}\right)\left(k_{3}-2 h_{1}-\mu t_{3}\right)}{\sigma \sqrt{t_{3}}} ;\left(I_{1}+I_{2}-I_{3}+I_{4}+I_{5}-I_{6}\right) \sqrt{\frac{t_{1}}{t_{2}}},\left(I_{1}-I_{2}-I_{3}+I_{4}-I_{5}-I_{6}\right) \sqrt{\frac{t_{1}}{t_{3}}}, \\
& \left.\left(I_{1}-I_{2}+I_{3}+I_{4}-I_{5}+I_{6}\right) \sqrt{\frac{t_{2}}{t_{3}}}\right]-\exp \left(\frac{2 \mu h_{2}}{\sigma^{2}}\right)
\end{aligned}
$$




$$
\begin{aligned}
& \cdot N_{3}\left[\frac{\left(I_{1}+I_{2}+I_{3}\right)\left(\left(I_{1}+I_{2}\right)\left(k_{1} \wedge h_{2}\right)+I_{3} k_{1}+\mu t_{1}\right)-\left(I_{4}+I_{5}+I_{6}\right)\left(\left(I_{4}+I_{5}\right)\left(k_{1} \vee h_{2}\right)+I_{6} k_{1}+\mu t_{1}\right)}{\sigma \sqrt{t_{1}}},\right. \\
& \frac{\left(I_{1}+I_{2}+I_{6}\right)\left(\left(I_{1}+I_{6}\right)\left(k_{2} \wedge h_{3}\right)+I_{2} k_{2}-2 h_{2}-\mu t_{2}\right)-\left(I_{3}+I_{4}+I_{5}\right)\left(\left(I_{3}+I_{4}\right)\left(k_{2} \vee h_{3}\right)+I_{5} k_{2}-2 h_{2}-\mu t_{2}\right)}{\sigma \sqrt{t_{2}}}, \\
& \frac{\left(I_{1}+I_{5}+I_{6}-I_{2}-I_{3}-I_{4}\right)\left(k_{3}-2 h_{2}-\mu t_{3}\right)}{\sigma \sqrt{t_{3}}} ;\left(-I_{1}-I_{2}+I_{3}-I_{4}-I_{5}+I_{6}\right) \sqrt{\frac{t_{1}}{t_{2}}},\left(-I_{1}+I_{2}+I_{3}-I_{4}+I_{5}+I_{6}\right) \sqrt{\frac{t_{1}}{t_{3}}}, \\
& \left.\left(I_{1}-I_{2}+I_{3}+I_{4}-I_{5}+I_{6}\right) \sqrt{\frac{t_{2}}{t_{3}}}\right]+\left(I_{2}+I_{3}+I_{5}+I_{6}\right) \exp \left(\frac{2 \mu h_{2}}{\sigma^{2}}\right) \\
& \cdot N_{3}\left[\frac{\left(I_{2}+I_{3}\right)\left(I_{2}\left(k_{1} \wedge h_{2}\right)+I_{3} h_{2}+\mu t_{1}\right)-\left(I_{5}+I_{6}\right)\left(I_{5}\left(k_{1} \vee h_{2}\right)+I_{6} h_{2}+\mu t_{1}\right)}{\sigma \sqrt{t_{1}}},\right. \\
& \frac{\left(I_{2}+I_{6}\right)\left(I_{2} h_{3}+I_{6}\left(k_{2} \wedge h_{3}\right)-2 h_{2}-\mu t_{2}\right)-\left(I_{3}+I_{5}\right)\left(I_{3}\left(k_{2} \vee h_{3}\right)+I_{5} h_{3}-2 h_{2}-\mu t_{2}\right)}{\sigma \sqrt{t_{2}}}, \\
& \frac{\left(I_{5}+I_{6}-I_{2}-I_{3}\right)\left(k_{3}-2 h_{2}-\mu t_{3}\right)}{\sigma \sqrt{t_{3}}} ;\left(-I_{1}-I_{2}+I_{3}-I_{4}-I_{5}+I_{6}\right) \sqrt{\frac{t_{1}}{t_{2}}},\left(-I_{1}+I_{2}+I_{3}-I_{4}+I_{5}+I_{6}\right) \sqrt{\frac{t_{1}}{t_{3}}}, \\
& \left.\left(I_{1}-I_{2}+I_{3}+I_{4}-I_{5}+I_{6}\right) \sqrt{\frac{t_{2}}{t_{3}}}\right]-\exp \left(\frac{2 \mu h_{3}}{\sigma^{2}}\right) \\
& \cdot N_{3}\left[\frac{\left(I_{1}+I_{2}+I_{3}\right)\left(\left(I_{1}+I_{2}\right)\left(k_{1} \wedge h_{2}\right)+I_{3} k_{1}+\mu t_{1}\right)-\left(I_{4}+I_{5}+I_{6}\right)\left(\left(I_{4}+I_{5}\right)\left(k_{1} \vee h_{2}\right)+I_{6} k_{1}+\mu t_{1}\right)}{\sigma \sqrt{t_{1}}},\right. \\
& \frac{\left(I_{1}+I_{2}+I_{6}\right)\left(\left(I_{1}+I_{6}\right)\left(k_{2} \wedge h_{3}\right)+I_{2} k_{2}+\mu t_{2}\right)-\left(I_{3}+I_{4}+I_{5}\right)\left(\left(I_{3}+I_{4}\right)\left(k_{2} \vee h_{3}\right)+I_{5} k_{2}+\mu t_{2}\right)}{\sigma \sqrt{t_{2}}}, \\
& \frac{\left(I_{1}+I_{5}+I_{6}-I_{2}-I_{3}-I_{4}\right)\left(k_{3}-2 h_{3}-\mu t_{3}\right)}{\sigma \sqrt{t_{3}}} ;\left(I_{1}+I_{2}-I_{3}+I_{4}+I_{5}-I_{6}\right) \sqrt{\frac{t_{1}}{t_{2}}},\left(-I_{1}+I_{2}+I_{3}-I_{4}+I_{5}+I_{6}\right) \sqrt{\frac{t_{1}}{t_{3}}}, \\
& \left.\left(-I_{1}+I_{2}-I_{3}-I_{4}+I_{5}-I_{6}\right) \sqrt{\frac{t_{2}}{t_{3}}}\right]+\left(I_{2}+I_{3}+I_{5}+I_{6}\right) \exp \left(\frac{2 \mu h_{3}}{\sigma^{2}}\right) \\
& \cdot N_{3}\left[\frac{\left(I_{2}+I_{3}\right)\left(I_{2}\left(k_{1} \wedge h_{2}\right)+I_{3} h_{2}+\mu t_{1}\right)-\left(I_{5}+I_{6}\right)\left(I_{5}\left(k_{1} \vee h_{2}\right)+I_{6} h_{2}+\mu t_{1}\right)}{\sigma \sqrt{t_{1}}},\right. \\
& \frac{\left(I_{2}+I_{6}\right)\left(I_{2} h_{3}+I_{6}\left(k_{2} \wedge h_{3}\right)+\mu t_{2}\right)-\left(I_{3}+I_{5}\right)\left(I_{3}\left(k_{2} \vee h_{3}\right)+I_{5} h_{3}+\mu t_{2}\right)}{\sigma \sqrt{t_{2}}}, \frac{\left(I_{5}+I_{6}-I_{2}-I_{3}\right)\left(k_{3}-2 h_{3}-\mu t_{3}\right)}{\sigma \sqrt{t_{3}}} \\
& \left.\left(I_{1}+I_{2}-I_{3}+I_{4}+I_{5}-I_{6}\right) \sqrt{\frac{t_{1}}{t_{2}}},\left(-I_{1}+I_{2}+I_{3}-I_{4}+I_{5}+I_{6}\right) \sqrt{\frac{t_{1}}{t_{3}}},\left(-I_{1}+I_{2}-I_{3}-I_{4}+I_{5}-I_{6}\right) \sqrt{\frac{t_{2}}{t_{3}}}\right] \\
& +\exp \left(\frac{2 \mu\left(h_{2}-h_{1}\right)}{\sigma^{2}}\right) \\
& \cdot N_{3}\left[\frac{\left(I_{1}+I_{2}+I_{3}\right)\left(\left(I_{1}+I_{2}\right)\left(k_{1} \wedge h_{2}\right)+I_{3} k_{1}-2 h_{1}+\mu t_{1}\right)-\left(I_{4}+I_{5}+I_{6}\right)\left(\left(I_{4}+I_{5}\right)\left(k_{1} \vee h_{2}\right)+I_{6} k_{1}-2 h_{1}+\mu t_{1}\right)}{\sigma \sqrt{t_{1}}},\right.
\end{aligned}
$$




$$
\begin{aligned}
& \frac{\left(I_{1}+I_{2}+I_{6}\right)\left(\left(I_{1}+I_{6}\right)\left(k_{2} \wedge h_{3}\right)+I_{2} k_{2}-2 h_{2}+2 h_{1}-\mu t_{2}\right)-\left(I_{3}+I_{4}+I_{5}\right)\left(\left(I_{3}+I_{4}\right)\left(k_{2} \vee h_{3}\right)+I_{5} k_{2}-2 h_{2}+2 h_{1}-\mu t_{2}\right)}{\sigma \sqrt{t_{2}}}, \\
& \frac{\left(I_{1}+I_{5}+I_{6}-I_{2}-I_{3}-I_{4}\right)\left(k_{3}-2 h_{2}+2 h_{1}-\mu t_{3}\right)}{\sigma \sqrt{t_{3}}} ;\left(-I_{1}-I_{2}+I_{3}-I_{4}-I_{5}+I_{6}\right) \sqrt{\frac{t_{1}}{t_{2}}},\left(-I_{1}+I_{2}+I_{3}-I_{4}+I_{5}+I_{6}\right) \sqrt{\frac{t_{1}}{t_{3}}}, \\
& \left.\left(I_{1}-I_{2}+I_{3}+I_{4}-I_{5}+I_{6}\right) \sqrt{\frac{t_{2}}{t_{3}}}\right]-\left(I_{2}+I_{3}+I_{5}+I_{6}\right) \exp \left(\frac{2 \mu\left(h_{2}-h_{1}\right)}{\sigma^{2}}\right) \\
& \cdot N_{3}\left[\frac{\left(I_{2}+I_{3}\right)\left(I_{2}\left(k_{1} \wedge h_{2}\right)+I_{3} h_{2}-2 h_{1}+\mu t_{1}\right)-\left(I_{5}+I_{6}\right)\left(I_{5}\left(k_{1} \vee h_{2}\right)+I_{6} h_{2}-2 h_{1}+\mu t_{1}\right)}{\sigma \sqrt{t_{1}}},\right. \\
& \frac{\left(I_{2}+I_{6}\right)\left(I_{2} h_{3}+I_{6}\left(k_{2} \wedge h_{3}\right)-2 h_{2}+2 h_{1}-\mu t_{2}\right)-\left(I_{3}+I_{5}\right)\left(I_{3}\left(k_{2} \vee h_{3}\right)+I_{5} h_{3}-2 h_{2}+2 h_{1}-\mu t_{2}\right)}{\sigma \sqrt{t_{2}}}, \\
& \frac{\left(I_{5}+I_{6}-I_{2}-I_{3}\right)\left(k_{3}-2 h_{2}+2 h_{1}-\mu t_{3}\right)}{\sigma \sqrt{t_{3}}} ;\left(-I_{1}-I_{2}+I_{3}-I_{4}-I_{5}+I_{6}\right) \sqrt{\frac{t_{1}}{t_{2}}},\left(I_{1}+I_{2}+I_{3}-I_{4}+I_{5}+I_{6}\right) \sqrt{\frac{t_{1}}{t_{3}}}, \\
& \left.\left(I_{1}-I_{2}+I_{3}+I_{4}-I_{5}+I_{6}\right) \sqrt{\frac{t_{2}}{t_{3}}}\right]+\exp \left(\frac{2 \mu\left(h_{3}-h_{1}\right)}{\sigma^{2}}\right) \\
& \cdot N_{3}\left[\frac{\left(I_{1}+I_{2}+I_{3}\right)\left(\left(I_{1}+I_{2}\right)\left(k_{1} \wedge h_{2}\right)+I_{3} k_{1}-2 h_{1}+\mu t_{1}\right)-\left(I_{4}+I_{5}+I_{6}\right)\left(\left(I_{4}+I_{5}\right)\left(k_{1} \vee h_{2}\right)+I_{6} k_{1}-2 h_{1}+\mu t_{1}\right)}{\sigma \sqrt{t_{1}}},\right. \\
& \frac{\left(I_{1}+I_{2}+I_{6}\right)\left(\left(I_{1}+I_{6}\right)\left(k_{2} \wedge h_{3}\right)+I_{2} k_{2}-2 h_{1}+\mu t_{2}\right)-\left(I_{3}+I_{4}+I_{5}\right)\left(\left(I_{3}+I_{4}\right)\left(k_{2} \vee h_{3}\right)+I_{5} k_{2}-2 h_{1}+\mu t_{2}\right)}{\sigma \sqrt{t_{2}}}, \\
& \frac{\left(I_{1}+I_{5}+I_{6}-I_{2}-I_{3}-I_{4}\right)\left(k_{3}-2 h_{3}+2 h_{1}-\mu t_{3}\right)}{\sigma \sqrt{t_{3}}} ;\left(I_{1}+I_{2}-I_{3}+I_{4}+I_{5}-I_{6}\right) \sqrt{\frac{t_{1}}{t_{2}}},\left(-I_{1}+I_{2}+I_{3}-I_{4}+I_{5}+I_{6}\right) \sqrt{\frac{t_{1}}{t_{3}}}, \\
& \left.\left(-I_{1}+I_{2}-I_{3}-I_{4}+I_{5}-I_{6}\right) \sqrt{\frac{t_{2}}{t_{3}}}\right]-\left(I_{2}+I_{3}+I_{5}+I_{6}\right) \exp \left(\frac{2 \mu\left(h_{3}-h_{1}\right)}{\sigma^{2}}\right) \\
& \cdot N_{3}\left[\frac{\left(I_{2}+I_{3}\right)\left(I_{2}\left(k_{1} \wedge h_{2}\right)+I_{3} h_{2}-2 h_{1}+\mu t_{1}\right)-\left(I_{5}+I_{6}\right)\left(I_{5}\left(k_{1} \vee h_{2}\right)+I_{6} h_{2}-2 h_{1}+\mu t_{1}\right)}{\sigma \sqrt{t_{1}}},\right. \\
& \frac{\left(I_{2}+I_{6}\right)\left(I_{2} h_{3}+I_{6}\left(k_{2} \wedge h_{3}\right)-2 h_{1}+\mu t_{2}\right)-\left(I_{3}+I_{5}\right)\left(I_{3}\left(k_{2} \vee h_{3}\right)+I_{5} h_{3}-2 h_{1}+\mu t_{2}\right)}{\sigma \sqrt{t_{2}}}, \\
& \frac{\left(I_{5}+I_{6}-I_{2}-I_{3}\right)\left(k_{3}-2 h_{3}+2 h_{1}-\mu t_{3}\right)}{\sigma \sqrt{t_{3}}} ;\left(I_{1}+I_{2}-I_{3}+I_{4}+I_{5}-I_{6}\right) \sqrt{\frac{t_{1}}{t_{2}}},\left(-I_{1}+I_{2}+I_{3}-I_{4}+I_{5}+I_{6}\right) \sqrt{\frac{t_{1}}{t_{3}}}, \\
& \left.\left(-I_{1}+I_{2}-I_{3}-I_{4}+I_{5}-I_{6}\right) \sqrt{\frac{t_{2}}{t_{3}}}\right]+\exp \left(\frac{2 \mu\left(h_{3}-h_{2}\right)}{\sigma^{2}}\right) \\
& \cdot N_{3}\left[\frac{\left(I_{1}+I_{2}+I_{3}\right)\left(\left(I_{1}+I_{2}\right)\left(k_{1} \wedge h_{2}\right)+I_{3} k_{1}-\mu t_{1}\right)-\left(I_{4}+I_{5}+I_{6}\right)\left(\left(I_{4}+I_{5}\right)\left(k_{1} \vee h_{2}\right)+I_{6} k_{1}-\mu t_{1}\right)}{\sigma \sqrt{t_{1}}},\right. \\
& \frac{\left(I_{1}+I_{2}+I_{6}\right)\left(\left(I_{1}+I_{6}\right)\left(k_{2} \wedge h_{3}\right)+I_{2} k_{2}-2 h_{2}+\mu t_{2}\right)-\left(I_{3}+I_{4}+I_{5}\right)\left(\left(I_{3}+I_{4}\right)\left(k_{2} \vee h_{3}\right)+I_{5} k_{2}-2 h_{2}+\mu t_{2}\right)}{\sigma \sqrt{t_{2}}},
\end{aligned}
$$




$$
\begin{aligned}
& \frac{\left(I_{1}+I_{5}+I_{6}-I_{2}-I_{3}-I_{4}\right)\left(k_{3}-2 h_{3}+2 h_{2}-\mu t_{3}\right)}{\sigma \sqrt{t_{3}}} ;\left(-I_{1}-I_{2}+I_{3}-I_{4}-I_{5}+I_{6}\right) \sqrt{\frac{t_{1}}{t_{2}}},\left(I_{1}-I_{2}-I_{3}+I_{4}-I_{5}-I_{6}\right) \sqrt{\frac{t_{1}}{t_{3}}}, \\
& \left.\left(-I_{1}+I_{2}-I_{3}-I_{4}+I_{5}-I_{6}\right) \sqrt{\frac{t_{2}}{t_{3}}}\right]-\left(I_{2}+I_{3}+I_{5}+I_{6}\right) \exp \left(\frac{2 \mu\left(h_{3}-h_{2}\right)}{\sigma^{2}}\right) \\
& \cdot N_{3}\left[\frac{\left(I_{2}+I_{3}\right)\left(I_{2}\left(k_{1} \wedge h_{2}\right)+I_{3} h_{2}-\mu t_{1}\right)-\left(I_{5}+I_{6}\right)\left(I_{5}\left(k_{1} \vee h_{2}\right)+I_{6} h_{2}-\mu t_{1}\right)}{\sigma \sqrt{t_{1}}},\right. \\
& \frac{\left(I_{2}+I_{6}\right)\left(I_{2} h_{3}+I_{6}\left(k_{2} \wedge h_{3}\right)-2 h_{2}+\mu t_{2}\right)-\left(I_{3}+I_{5}\right)\left(I_{3}\left(k_{2} \vee h_{3}\right)+I_{5} h_{3}-2 h_{2}+\mu t_{2}\right)}{\sigma \sqrt{t_{2}}}, \\
& \frac{\left(I_{5}+I_{6}-I_{2}-I_{3}\right)\left(k_{3}-2 h_{3}+2 h_{2}-\mu t_{3}\right)}{\sigma \sqrt{t_{3}}} ;\left(-I_{1}-I_{2}+I_{3}-I_{4}-I_{5}+I_{6}\right) \sqrt{\frac{t_{1}}{t_{2}}},\left(I_{1}-I_{2}-I_{3}+I_{4}-I_{5}-I_{6}\right) \sqrt{\frac{t_{1}}{t_{3}}}, \\
& \left.\left(-I_{1}+I_{2}-I_{3}-I_{4}+I_{5}-I_{6}\right) \sqrt{\frac{t_{2}}{t_{3}}}\right]-\exp \left(\frac{2 \mu\left(h_{3}-h_{2}+h_{1}\right)}{\sigma^{2}}\right) \\
& \cdot N_{3}\left[\frac{\left(I_{1}+I_{2}+I_{3}\right)\left(\left(I_{1}+I_{2}\right)\left(k_{1} \wedge h_{2}\right)+I_{3} k_{1}-2 h_{1}-\mu t_{1}\right)-\left(I_{4}+I_{5}+I_{6}\right)\left(\left(I_{4}+I_{5}\right)\left(k_{1} \vee h_{2}\right)+I_{6} k_{1}-2 h_{1}-\mu t_{1}\right)}{\sigma \sqrt{t_{1}}},\right. \\
& \frac{\left(I_{1}+I_{2}+I_{6}\right)\left(\left(I_{1}+I_{6}\right)\left(k_{2} \wedge h_{3}\right)+I_{2} k_{2}-2 h_{2}+2 h_{1}+\mu t_{2}\right)-\left(I_{3}+I_{4}+I_{5}\right)\left(\left(I_{3}+I_{4}\right)\left(k_{2} \vee h_{3}\right)+I_{5} k_{2}-2 h_{2}+2 h_{1}+\mu t_{2}\right)}{\sigma \sqrt{t_{2}}}, \\
& \frac{\left(I_{1}+I_{5}+I_{6}-I_{2}-I_{3}-I_{4}\right)\left(k_{3}-2 h_{3}+2 h_{2}-2 h_{1}-\mu t_{3}\right)}{\sigma \sqrt{t_{3}}} ;\left(-I_{1}-I_{2}+I_{3}-I_{4}-I_{5}+I_{6}\right) \sqrt{\frac{t_{1}}{t_{2}}},\left(I_{1}-I_{2}-I_{3}+I_{4}-I_{5}-I_{6}\right) \\
& \left.\cdot \sqrt{\frac{t_{1}}{t_{3}}},\left(-I_{1}+I_{2}-I_{3}-I_{4}+I_{5}-I_{6}\right) \sqrt{\frac{t_{2}}{t_{3}}}\right]+\left(I_{2}+I_{3}+I_{5}+I_{6}\right) \exp \left(\frac{2 \mu\left(h_{3}-h_{2}+h_{1}\right)}{\sigma^{2}}\right) \\
& \cdot N_{3}\left[\frac{\left(I_{2}+I_{3}\right)\left(I_{2}\left(k_{1} \wedge h_{2}\right)+I_{3} h_{2}-2 h_{1}-\mu t_{1}\right)-\left(I_{5}+I_{6}\right)\left(I_{5}\left(k_{1} \vee h_{2}\right)+I_{6} h_{2}-2 h_{1}-\mu t_{1}\right)}{\sigma \sqrt{t_{1}}},\right. \\
& \frac{\left(I_{2}+I_{6}\right)\left(I_{2} h_{3}+I_{6}\left(k_{2} \wedge h_{3}\right)-2 h_{2}+2 h_{1}+\mu t_{2}\right)-\left(I_{3}+I_{5}\right)\left(I_{3}\left(k_{2} \vee h_{3}\right)+I_{5} h_{3}-2 h_{2}+2 h_{1}+\mu t_{2}\right)}{\sigma \sqrt{t_{2}}}, \\
& \frac{\left(I_{5}+I_{6}-I_{2}-I_{3}\right)\left(k_{3}-2 h_{3}+2 h_{2}-2 h_{1}-\mu t_{3}\right)}{\sigma \sqrt{t_{3}}} ;\left(-I_{1}-I_{2}+I_{3}-I_{4}-I_{5}+I_{6}\right) \sqrt{\frac{t_{1}}{t_{2}}},\left(I_{1}-I_{2}-I_{3}+I_{4}-I_{5}-I_{6}\right) \sqrt{\frac{t_{1}}{t_{3}}}, \\
& \left.\left(-I_{1}+I_{2}-I_{3}-I_{4}+I_{5}-I_{6}\right) \sqrt{\frac{t_{2}}{t_{3}}}\right]
\end{aligned}
$$

where

$I_{1}=1$ if $P_{[\ldots]}=P_{[M M M]}$ and zero otherwise; $I_{2}=1$ if $P_{[\ldots]}=P_{[M M m]}$ and zero otherwise;

$I_{3}=1$ if $P_{[\ldots]}=P_{[\mathrm{Mmm}]}$ and zero otherwise; $I_{4}=1$ if $P_{[\cdots]}=P_{[\mathrm{mmm}]}$ and zero otherwise;

$I_{5}=1$ if $P_{[\ldots]}=P_{[m m M]}$ and zero otherwise, $I_{6}=1$ if $P_{[\cdots]}=P_{[m M M]}$ and zero otherwise.
And the function $N_{3}\left[x_{1}, x_{2}, x_{3} ; \rho_{12}, \rho_{13}, \rho_{23}\right]$ is the cumulative distribution function of three correlated standard normal random variables $X_{1}, X_{2}$, and $X_{3}$ with upper bounds $\left(x_{1}, x_{2}, x_{3}\right) \in$ $\mathbb{R}^{3}$ and correlation coefficients $\rho_{12}$ between $X_{1}$ and $X_{2}, \rho_{13}$ between $X_{1}$ and $X_{3}$, and $\rho_{23}$ between $X_{2}$ and $X_{3}$; the numerical evaluation of this function can be carried out with double precision and computational time of approximately 0.01 second using the algorithm by Genz [17]. 
Proof of Proposition 1. $X\left(t_{1}\right), X\left(t_{2}\right)$, and $X\left(t_{3}\right)$ are absolutely continuous random variables. Moreover, $\{X(t), t \geq 0\}$ is a Markov process. Hence, by applying the law of total probability and by conditioning, we have

$$
\begin{aligned}
P_{[\ldots]} & \left(h_{1}, h_{2}, h_{3}, k_{1}, k_{2}, k_{3}, t_{1}, t_{2}, t_{3}\right)=\int_{a_{1}}^{a_{2}} \int_{a_{3}}^{a_{4}} \int_{a_{5}}^{a_{6}} \mathbb{P}\left(X\left(t_{1}\right) \in d x_{1}, X\left(t_{2}\right) \in d x_{2}, X\left(t_{3}\right) \in d x_{3}\right) \\
& \cdot\left(\mathbb{P}\left(\sup _{0 \leq t \leq t_{1}} X(t)<h_{1} \mid X\left(t_{1}\right)\right)\left(I_{1}+I_{2}+I_{3}\right)+\mathbb{P}\left(\inf _{0 \leq t \leq t_{1}} X(t)>h_{1} \mid X\left(t_{1}\right)\right)\left(I_{4}+I_{5}+I_{6}\right)\right) \\
& \cdot\left(\mathbb{P}\left(\sup _{t_{1} \leq t \leq t_{2}} X(t)<h_{2} \mid X\left(t_{1}\right), X\left(t_{2}\right)\right)\left(I_{1}+I_{2}+I_{6}\right)+\mathbb{P}\left(\inf _{t_{1} \leq t \leq t_{2}} X(t)>h_{2} \mid X\left(t_{1}\right), X\left(t_{2}\right)\right)\left(I_{3}+I_{4}+I_{5}\right)\right) \\
& \cdot\left(\mathbb{P}\left(\sup _{t_{2} \leq t \leq t_{3}} X(t)<h_{3} \mid X\left(t_{2}\right), X\left(t_{3}\right)\right)\left(I_{1}+I_{5}+I_{6}\right)+\mathbb{P}\left(\inf _{t_{2} \leq t \leq t_{3}} X(t)>h_{3} \mid X\left(t_{2}\right), X\left(t_{3}\right)\right)\left(I_{2}+I_{3}+I_{4}\right)\right) d x_{3} d x_{2} d x_{1},
\end{aligned}
$$

where $a_{1}=(-\infty)\left(I_{1}+I_{2}+I_{3}\right)+k_{1}\left(I_{4}+I_{5}+I_{6}\right)$, $a_{2}=k_{1}\left(I_{1}+I_{2}+I_{3}\right)+(\infty)\left(I_{4}+I_{5}+I_{6}\right), a_{3}=(-\infty)\left(I_{1}+\right.$ $\left.I_{2}+I_{6}\right)+k_{2}\left(I_{3}+I_{4}+I_{5}\right), a_{4}=k_{2}\left(I_{1}+I_{2}+I_{6}\right)+(\infty)\left(I_{3}+\right.$ $\left.I_{4}+I_{5}\right), a_{5}=(-\infty)\left(I_{1}+I_{5}+I_{6}\right)+k_{3}\left(I_{2}+I_{3}+I_{4}\right)$, and $a_{6}=k_{3}\left(I_{1}+I_{5}+I_{6}\right)+(\infty)\left(I_{2}+I_{3}+I_{4}\right)$ and $X\left(t_{1}\right), X\left(t_{2}\right)$, and $X\left(t_{3}\right)$ are jointly normal random variables and each pairwise correlation coefficient is equal to $\sqrt{t_{i} / t_{j}}, \forall(i, j) \epsilon$ $\{1,2,3\}, i<j$. Besides, the distribution of the maximum (or the minimum) of a Brownian motion with drift on

$$
\begin{aligned}
P_{[\cdots]} & \left(h_{1}, h_{2}, h_{3}, k_{1}, k_{2}, k_{3}, t_{1}, t_{2}, t_{3}\right) \\
& =\int_{a_{1}}^{a_{2}} \int_{a_{3}}^{a_{4}} \int_{a_{5}}^{a_{6}} \frac{\exp \left(-\bar{x}_{1}^{2} / 2-\left(1 / 2 \lambda_{2 \mid 1}^{2}\right)\left(\bar{x}_{2}-\rho_{1.2} \bar{x}_{1}\right)^{2}-\left(1 / 2 \lambda_{3 \mid 1.2}^{2}\right)\left(\bar{x}_{3}-\rho_{1.3} \bar{x}_{1}-\theta_{2.3 \mid 1}\left(\left(\bar{x}_{2}-\rho_{1.2} \bar{x}_{1}\right) / \lambda_{2 \mid 1}\right)\right)^{2}\right)}{\sqrt{8 \pi^{3}} \lambda_{2 \mid 1} \lambda_{3 \mid 1.2} \sigma^{3} \sqrt{t_{1} t_{2} t_{3}}}\left(1-\exp \left(\frac{2 h_{1}\left(x_{1}-h_{1}\right)}{\sigma^{2} t_{1}}\right)\right) \\
& \cdot\left(1-\exp \left(\frac{2\left(h_{2}-x_{1}\right)\left(x_{2}-h_{2}\right)}{\sigma^{2}\left(t_{2}-t_{1}\right)}\right)\right)\left(1-\exp \left(\frac{2\left(h_{3}-x_{2}\right)\left(x_{3}-h_{3}\right)}{\sigma^{2}\left(t_{3}-t_{2}\right)}\right)\right) d x_{3} d x_{2} d x_{1},
\end{aligned}
$$

a closed time interval, conditional on the two endpoints, is given by

$$
\begin{aligned}
& \mathbb{P}\left(\sup _{t_{i} \leq t \leq t_{j}} X(t)<h_{j} \mid X\left(t_{i}\right) \in d x_{i}, X\left(t_{j}\right) \in d x_{j}\right) \\
& \quad=\mathbb{P}\left(\inf _{t_{i} \leq t \leq t_{j}} X(t)>h_{j} \mid X\left(t_{i}\right) \in d x_{i}, X\left(t_{j}\right) \in d x_{j}\right) \\
& \quad=1-\exp \left(\frac{2\left(h_{j}-x_{i}\right)\left(x_{j}-h_{j}\right)}{\sigma^{2}\left(t_{j}-t_{i}\right)}\right) .
\end{aligned}
$$

Thus, expanding the trivariate normal probability density function, using (5) and substituting into (4), we obtain

where

$$
\begin{aligned}
\bar{x}_{1} & =\frac{x_{1}-\mu t_{1}}{\sigma \sqrt{t_{1}}}, \\
\bar{x}_{2} & =\frac{x_{2}-\mu t_{2}}{\sigma \sqrt{t_{2}}} \\
\bar{x}_{3} & =\frac{x_{3}-\mu t_{3}}{\sigma \sqrt{t_{3}}} \\
\rho_{1.2} & =\sqrt{\frac{t_{1}}{t_{2}}} \\
\rho_{1.3} & =\sqrt{\frac{t_{1}}{t_{3}}}
\end{aligned}
$$

$$
\begin{aligned}
\rho_{2.3} & =\sqrt{\frac{t_{2}}{t_{3}}}, \\
\lambda_{2 \mid 1} & =\sqrt{1-\rho_{1.2}^{2}}, \\
\theta_{2.3 \mid 1} & =\frac{\rho_{2.3}-\rho_{1.2} \rho_{1.3}}{\lambda_{2 \mid 1}}, \\
\lambda_{3 \mid 1.2} & =\sqrt{1-\rho_{1.3}^{2}-\theta_{2.3 \mid 1}^{2}} .
\end{aligned}
$$

It is possible to solve this triple integral in closed form as a linear combination of sixteen trivariate standard normal cumulative distribution functions and this yields Proposition 1 . The details are quite cumbersome, so they are omitted. However, 
it is easy to verify that a Monte Carlo simulation will produce numbers that slowly converge to the exact values provided by Proposition 1 whatever the parameters considered. It is highly recommended to use a conditional Monte Carlo approach inasmuch as the latter enables approximating the value of the integral in (6) by randomly drawing three and only three values of the process $\{X(t), t \geq 0\}$ at each run, without having to discretize the time interval; for some background on the conditional Monte Carlo approach, also known as Brownian Bridge Monte Carlo, the reader is referred to Dagpunar [18]. After implementing conditional Monte Carlo and drawing random numbers from the Mersenne Twister generator, we observed that at least 20,000,000 simulations were required to be sure to attain a minimum level of $10^{-5}$ convergence with the values provided by Proposition 1 . These empirical findings were obtained out of a sample of 1,500 lists of randomly drawn parameters used as inputs to Proposition 1. They are reported in more detail in Table 1.

Before turning to Section 3, one may notice that the following combinations of steps are not handled by Proposition 1:

$$
\begin{aligned}
& P_{[m M m]}\left(h_{1}, h_{2}, h_{3}, k_{1}, k_{2}, k_{3}, t_{1}, t_{2}, t_{3}\right) \triangleq \mathbb{P}\left(\inf _{0 \leq t \leq t_{1}} X(t)\right. \\
& \quad \geq h_{1}, X\left(t_{1}\right) \geq k_{1}, \sup _{t_{1} \leq t \leq t_{2}} X(t) \leq h_{2}, X\left(t_{2}\right) \leq k_{2}, \inf _{t_{2} \leq t \leq t_{3}} X(t) \\
& \left.\quad \geq h_{3}, X\left(t_{3}\right) \geq k_{3}\right) \\
& h_{1} \in \mathbb{R}_{-},\left(h_{2}, h_{3}, k_{1}, k_{2}, k_{3}\right) \in \mathbb{R}^{5}, k_{1} \geq h_{1}, k_{2} \leq h_{2}, k_{3} \geq h_{3}, \\
& P_{[M m M]}\left(h_{1}, h_{2}, h_{3}, k_{1}, k_{2}, k_{3}, t_{1}, t_{2}, t_{3}\right) \triangleq \mathbb{P}\left(\sup _{0 \leq t \leq t_{1}} X(t)\right. \\
& \quad \leq h_{1}, X\left(t_{1}\right) \leq k_{1}, \inf _{t_{1} \leq t \leq t_{2}} X(t) \geq h_{2}, X\left(t_{2}\right) \geq k_{2}, \sup _{t_{2} \leq t \leq t_{3}} X(t) \\
& \left.\quad \leq h_{3}, X\left(t_{3}\right) \leq k_{3}\right) \\
& h_{1} \in \mathbb{R}_{+},\left(h_{2}, h_{3}, k_{1}, k_{2}, k_{3}\right) \in \mathbb{R}^{5}, k_{1} \leq h_{1}, k_{2} \geq h_{2}, k_{3} \leq h_{3} .
\end{aligned}
$$

This is not because (8) and (9) are more difficult to obtain using the method outlined in the proof of Proposition 1 but simply because the resulting formulae involve twice as many multivariate distribution functions as in the statement of Proposition 1, which would make the latter very bulky. Closed form formulae for (8) and (9) are available from the author upon request.

\section{Numerical Experiments}

Let us consider a geometric Brownian motion driven by

$$
d S(t)=\nu S(t) d t+\sigma S(t) d B(t)
$$

where $v$ is a constant, $\sigma$ is a positive constant, and $\{B(t), t \geq$ $0\}$ is a standard Brownian motion.

Without loss of generality, this process is assumed to start at level $S(0)=100$ at time $t_{0}$. We first deal with sequences of maxima or minima, that is, $P_{[M M M]}$-type and $P_{[\mathrm{mmm}]^{-}}$ cumulative distribution functions. We are interested in the following survival probabilities:

$$
\begin{array}{r}
\mathbb{P}\left(\sup _{0 \leq t \leq t_{1}} S(t) \leq H_{1}, \sup _{t_{1} \leq t \leq t_{2}} S(t) \leq H_{2}, \sup _{t_{2} \leq t \leq t_{3}} S(t) \leq H_{3}\right), \\
H_{1}>S(0), \\
\mathbb{P}\left(\inf _{0 \leq t \leq t_{1}} S(t) \geq H_{1}, \inf _{t_{1} \leq t \leq t_{2}} S(t) \geq H_{2}, \inf _{t_{2} \leq t \leq t_{3}} S(t) \geq H_{3}\right), \\
H_{1}<S(0) .
\end{array}
$$

An elementary application of Ito's lemma to $\ln (S(t) / S(0))$ shows that (9) and (10) can be obtained by computing

$$
\begin{aligned}
& \mathbb{P}\left(\sup _{0 \leq t \leq t_{1}} X(t) \leq h_{1}, X\left(t_{1}\right) \leq h_{1}, \sup _{t_{1} \leq t \leq t_{2}} X(t)\right. \\
& \left.\quad \leq h_{2}, X\left(t_{2}\right) \leq h_{2}, \sup _{t_{2} \leq t \leq t_{3}} X(t) \leq h_{3}, X\left(t_{3}\right) \leq h_{3}\right), \\
& \mathbb{P}\left(\inf _{0 \leq t \leq t_{1}} X(t) \geq h_{1}, X\left(t_{1}\right) \geq h_{1}, \inf _{t_{1} \leq t \leq t_{2}} X(t)\right. \\
& \left.\quad \geq h_{2}, X\left(t_{2}\right) \geq h_{2}, \inf _{t_{2} \leq t \leq t_{3}} X(t) \geq h_{3}, X\left(t_{3}\right) \geq h_{3}\right),
\end{aligned}
$$

where $h_{i}=\ln \left(H_{i} / S(0)\right)$ and $X(t)$ is driven by

$$
d X(t)=\mu d t+\sigma d B(t), \quad \mu=v-\frac{\sigma^{2}}{2} .
$$

Thus, Proposition 1 can be used to compute exact survival probabilities of the geometric Brownian motion $\{S(t), t \geq 0\}$.

The boundaries under consideration are said to "widen" on a given time interval when the current step is located farther from the initial process value than the previous step. Likewise, the boundaries are said to "shrink" on a given time interval when the current step is nearer to the initial process value than the previous step. Our objective is threefold:

(i) To measure the effect of widening the boundary at one or two steps, compared with keeping a fixed boundary until maturity.

(ii) To measure the effect of ordering the steps of the boundary in different ways; in particular, we compare survival probabilities when the farthest step is located in the last time interval and the nearest step is located in the first time interval.

(iii) To compare, conversely, survival probabilities when the boundary is upward and when it is downward.

For the latter comparison to be unbiased or neutral, we select upward and downward steps whose distances to the origin $S(0)$ are the same. Additionally, the probability of not hitting a boundary is a function of the deterministic part of the stochastic differential equation driving the process: a positive drift coefficient $v$ increases the chances of hitting an upward 
TABLE 1: Numerical comparison between Proposition 1 and conditional Monte Carlo (CMC) approximation ${ }^{1}$.

\begin{tabular}{|c|c|c|c|c|}
\hline & Proposition 1 & $\begin{array}{c}\mathrm{CMC} \\
500,000\end{array}$ & $\begin{array}{c}\mathrm{CMC} \\
5,000,000\end{array}$ & $\begin{array}{c}\mathrm{CMC} \\
20,000,000\end{array}$ \\
\hline Average divergence (\%) & 0 & 0.29874 & 0.021496 & 0.00046 \\
\hline Maximum divergence (\%) & 0 & 0.84231 & 0.13884 & 0.087 \\
\hline Computational time (seconds) & 0.59 & 24.57 & 239.26 & 953.42 \\
\hline
\end{tabular}

${ }^{1}$ The average divergence is the average of the absolute values of the differences between Proposition 1 and CMC divided by Proposition 1 out of a sample of 1,500 lists of randomly drawn parameters.

The maximum divergence is the maximum of the absolute values of the differences between Proposition 1 and CMC divided by Proposition 1 out of a sample of 1,500 lists of randomly drawn parameters.

Computational time is measured on an i3 $2.5 \mathrm{Ghz}$ processor.

TABLE 2: Survival probability for various sequences of upward absorbing steps as a function of volatility ${ }^{2}$.

\begin{tabular}{lccc}
\hline & $\sigma=18 \%$ & $\sigma=36 \%$ & $\sigma=64 \%$ \\
\hline$H_{1}=H_{2}=H_{3}=120$ & 0.73861288 & 0.46868224 & 0.31769242 \\
$H_{1}=120, H_{2}=120$, & 0.83295259 & 0.53187603 & 0.34607144 \\
$H_{3}=140$ & & & \\
$H_{1}=140, H_{2}=120$, & 0.74289172 & 0.51226639 & 0.40804930 \\
$H_{3}=120$ & & & \\
$H_{1}=120, H_{2}=140$, & 0.75896593 & 0.50006440 & 0.33948898 \\
$H_{3}=120$ & & & \\
$H_{1}=120, H_{2}=126$, & 0.88521655 & 0.55858123 & 0.35488207 \\
$H_{3}=134$ & & & \\
$H_{1}=134, H_{2}=126$, & 0.76627014 & 0.54546490 & 0.41461240 \\
$H_{3}=120$ & & & \\
$H_{1}=126, H_{2}=120$, & 0.83167791 & 0.55637189 & 0.38677980 \\
$H_{3}=134$ & & & \\
\hline
\end{tabular}

${ }^{2}$ All the values reported in this table are obtained by using Proposition 1 with $S(0)=100, v=3 \%, t_{1}=0.25, t_{2}=0.5, t_{3}=0.75, K_{1}=H_{1}, K_{2}=H_{2}$, and $K_{3}=H_{3}$.

boundary, while a negative drift coefficient $v$ increases the chances of hitting a downward boundary. That is why we set symmetric values of $\nu$ of $3 \%$ and $-3 \%$ when examining sequences of maxima and sequences of minima, respectively.

Three levels of the dispersion coefficient $\sigma$ are selected: $18 \%$ (defining a "low volatility regime"), 36\% (defining an "intermediate volatility regime"), and 64\% (defining a "high volatility regime").

Numerical results are reported in Tables 2 and 3.

First, it can be noticed that the increase in the survival probability entailed by a widening of the boundary is not symmetric whether one deals with an upward or a downward boundary. In a low volatility regime, the probability of remaining above a downward boundary is higher than the probability of remaining below an upward boundary. The reverse holds in a high volatility regime. In the intermediate volatility regime, the result depends on the order of the steps of the boundary.

It must be stressed that not all these observations are intuitive. Recall that the drift and the dispersion coefficients
TABLE 3: Survival probability for various sequences of downward absorbing steps as a function of volatility ${ }^{3}$.

\begin{tabular}{lccc}
\hline & $\sigma=18 \%$ & $\sigma=36 \%$ & $\sigma=64 \%$ \\
\hline$H_{1}=H_{2}=H_{3}=80$ & 0.7939442 & 0.4478266 & 0.2294535 \\
$H_{1}=80, H_{2}=80$, & 0.8918561 & 0.5501897 & 0.2833042 \\
$H_{3}=60$ & & & \\
$H_{1}=60, H_{2}=80$, & 0.7951798 & 0.4722809 & 0.2852277 \\
$H_{3}=80$ & & & \\
$H_{1}=80, H_{2}=60$, & 0.8070047 & 0.4779041 & 0.2511610 \\
$H_{3}=80$ & & & \\
$H_{1}=80, H_{2}=74$, & 0.9588887 & 0.6025832 & 0.2968822 \\
$H_{3}=66$ & & & \\
$H_{1}=66, H_{2}=74$, & 0.8103539 & 0.5135717 & 0.3087369 \\
$H_{3}=80$ & & & \\
$H_{1}=74, H_{2}=80$, & 0.8926716 & 0.5667168 & 0.3125188 \\
$H_{3}=66$ & & &
\end{tabular}

${ }^{3}$ All the values reported in this table are obtained by using Proposition 1 with $S(0)=100, v=-3 \%, t_{1}=0.25, t_{2}=0.5, t_{3}=0.75, K_{1}=H_{1}, K_{2}=H_{2}$, and $K_{3}=H_{3}$.

of a geometric Brownian motion such as $\{S(t), t \geq 0\}$ are proportional to $S(t)$ at any given $t \geq 0$, so that the drift coefficient of $\{\ln S(t) / S(0), t \geq 0\}$ is equal to $\mu \triangleq v-\sigma^{2} / 2$. When $\sigma=64 \%$, we have $\mu=-17.48 \%$ if $v=3 \%$ as in Table 2 and $\mu=-23.48 \%$ if $v=-3 \%$ as in Table 3. The deterministic part of the instantaneous dynamics thus drives the process downward in the high volatility regime, so that it decreases the likelihood of crossing an upward boundary and increases the likelihood of crossing a downward boundary. It is therefore not surprising to observe that the probability of remaining below an upward boundary is higher than that of remaining above a downward boundary in the high volatility regime, all the more so as $\mu$ is more negative in Table 3 than in Table 2. However, the observation that one can make in the low volatility regime may seem counter-intuitive. Indeed, when $\sigma=18 \%$, we have $\mu=1.38 \%$ if $v=3 \%$ and $\mu=-4.62 \%$ if $v=-3 \%$. The deterministic part of the instantaneous dynamics thus drives the process upward in Table 2, which has a negative effect on the probability of survival; but the process is driven downward in Table 3, which 
also creates a negative effect on the probability of survival and at a faster rate than that with which the process is driven upward in Table 2. Hence, from an intuitive point of view, one would expect the probability of remaining below an upward boundary to be higher than that of remaining above a downward boundary in the low volatility regime too.

Second, one can observe that widening the boundary has a much more significant impact when the farthest step is located in the last time interval if volatility is low, whether one deals with an upward or a downward boundary, while the reverse is true in a high volatility regime; that is, the probability that the process will have survived at time $t_{3}$ is higher when the farthest step is set in the first time interval. This phenomenon can be explained by the fact that the chances of hitting the boundary are already substantial early on in the life of the process when volatility is high, so that widening the boundary at the first time interval exerts a "strong" positive impact on the probability of survival, whereas the risk of absorption only gradually increases over time when volatility is low, so that widening the boundary at an early stage has less effect on the probability of survival. However, the magnitude of this phenomenon in a high volatility regime is much larger when the boundary is upward rather than downward, which suggests that when volatility is high, the risk of hitting the boundary at an early stage of the process is greater when the boundary is upward. Interestingly enough, the probabilities of survival are very close in Table 3 when the farthest step of the two-step boundary is located in the first or in the last time interval and volatility is high.

Another noticeable fact is the fact that the chances of survival rise when setting two gradually increasing or decreasing steps rather than one much higher or much lower step whatever the level of volatility. More precisely, the survival probability with a two-step boundary is lower than that with a three-step boundary whether the boundary is widening or shrinking even as we consider an identical weighted average of the steps in both cases; in other words, it is "safer" to go through the set of steps $\{120,126,134\}$ rather than through the set of steps $\{120,120,140\}$ and it is "safer" to go through the set of steps $\{134,126,120\}$ rather than through the set of steps $\{140,120,120\}$, although the average (weighted by each time interval) is $133+1 / 3$ in both sets; similarly, it is safer to go through the set of steps $\{80,74,66\}$ rather than through the set of steps $\{80,80,60\}$ and it is safer to go through the set of steps $\{66,74,80\}$ rather than through the set of steps $\{60,80,80\}$, although the weighted average is $73+1 / 3$ in both sets. The differences between survival probabilities as a function of the number of steps in the boundary are smaller when volatility is high than when it is intermediate or low.

These observations suggest that we should use Proposition 1 to compute the probability that the maximum (or the minimum) reached by the process $\{S(t), t \geq 0\}$ will lie in the first, the second, or the third time interval, as a function of volatility, considering three time intervals of identical length (otherwise, it is easily anticipated that any extremum will more likely be reached in any time interval (i.e., significantly larger than the others)). The probability that the maximum will lie in the first time interval can be obtained by computing

$$
\int_{0}^{\infty} \int_{0}^{x_{1}} \int_{0}^{x_{1}} \mathbb{P}\left(\sup _{0 \leq t \leq t_{1}} X(t) \in d x_{1}, \sup _{t_{1} \leq t \leq t_{2}} X(t) \in d x_{2}, \sup _{t_{2} \leq t \leq t_{3}} X(t) \in d x_{3}\right) d x_{3} d x_{2} d x_{1} .
$$

The trivariate density function inside this integral is obtained by differentiating Proposition 1 three times. This operation is tedious but straightforward and can be dealt with painlessly by using standard scientific computing software. The probabilities that the maximum will lie in the second or in the third time interval can be computed likewise in an obvious manner. Numerical results are reported in Table 4, using parameters consistent with Table 2 . The quantity $S^{*}$ is defined as the global maximum; that is,

$$
\begin{aligned}
S^{*} & \triangleq \max \left(\sup _{0 \leq t \leq t_{1}} S(t), \sup _{t_{1} \leq t \leq t_{2}} S(t), \sup _{t_{2} \leq t \leq t_{3}} S(t)\right) \\
& =\sup _{0 \leq t \leq t_{3}} S(t) .
\end{aligned}
$$

Conditional expected values of $S^{*}$, obtained by Monte Carlo simulation, are also reported.

We can see that, whatever the level of volatility, the probability that $S^{*}$ is reached between $t_{1}$ and $t_{2}$, that is, in the middle time interval, is approximately the same. It is also always the least likely outcome. In contrast, the probability that $S^{*}$ is reached between $t_{0}$ and $t_{1}$, that is, in the first time interval, steadily increases with $\sigma$, while the probability that $S^{*}$ is reached between $t_{2}$ and $t_{3}$, that is, in the last time interval, steadily decreases with $\sigma$. When volatility is low, the most likely outcome is that $S^{*}$ will be reached in the last time interval. This result is quite intuitive, as low volatility implies that it takes time for the process to hit large positive values and that the chances that the process will plummet at an early stage are thin. When volatility is high, the most likely outcome is that $S^{*}$ will be reached in the first time interval. This could be because of the fact that as volatility rises the likelihood that the process $\{S(t), t \geq 0\}$ will hit lower and lower values over time increases, so that an increasingly smaller fraction of paths will have enough time to make the journey back to highly positive territory. This explanation is supported by the distribution of the location of $S^{*}$ in space. Indeed, Table 4 shows that when $S^{*}$ is reached in the first time interval, the expected value of $S^{*}$ is always smaller than that when $S^{*}$ is reached in the second time interval or in the third time interval, whatever the level of volatility, which implies that the occurrence of the event $\left\{S^{*}=\sup _{0 \leq t \leq t_{1}} S(t)\right\}$ 
TABLE 4: Distribution of the global maximum over the various time intervals as a function of volatility ${ }^{4}$.

\begin{tabular}{llll}
\hline & $\sigma=18 \%$ & $\sigma=36 \%$ & $\sigma=64 \%$ \\
\hline $\mathbb{P}\left(S^{*}=\sup _{0 \leq t \leq t_{1}} S(t)\right)$ & 0.37121 & 0.41847 & 0.46731 \\
\hline $\mathbb{E}\left[S^{*} \mid S^{*}=\sup _{0 \leq t \leq t_{1}} S(t)\right]$ & 106.111 & 112.038 & 121.318 \\
\hline $\mathbb{P}\left(S^{*}=\sup _{t_{1} \leq t \leq t_{2}} S(t)\right)$ & 0.21601 & 0.21589 & 0.21306 \\
\hline $\mathbb{E}\left[S^{*} \mid S^{*}=\sup _{t_{1} \leq t \leq t_{2}} S(t)\right]$ & 115.013 & 130.861 & 158.376 \\
\hline $\mathbb{P}\left(S^{*}=\sup _{t_{2} \leq t \leq t_{3}} S(t)\right)$ & 0.41276 & 0.36562 & 0.31971 \\
\hline $\mathbb{E}\left[S^{*} \mid S^{*}=\sup _{t_{2} \leq t \leq t_{3}} S(t)\right]$ & 120.625 & 142.826 & 182.615 \\
\hline
\end{tabular}

${ }^{4}$ The parameters used in this table are as follows: $S(0)=100, v=3 \%, t_{1}=$ $0.25, t_{2}=0.5$, and $t_{3}=0.75$.

TABLE 5: Distribution of the global maximum over the various time intervals as a function of volatility on a longer time horizon than that in Table $4^{5}$.

\begin{tabular}{llll}
\hline & $\sigma=18 \%$ & $\sigma=36 \%$ & $\sigma=64 \%$ \\
\hline $\mathbb{P}\left(S^{*}=\sup _{0 \leq t \leq t_{1}} S(t)\right)$ & 0.35094 & 0.44517 & 0.54329 \\
\hline $\mathbb{E}\left[S^{*} \mid S^{*}=\sup _{0 \leq t \leq t_{1}} S(t)\right]$ & 112.86 & 124.826 & 143.49 \\
\hline $\mathbb{P}\left(S^{*}=\sup _{t_{1} \leq t \leq t_{2}} S(t)\right)$ & 0.21522 & 0.21498 & 0.20364 \\
\hline $\mathbb{E}\left[S^{*} \mid S^{*}=\sup _{t_{1} \leq t \leq t_{2}} S(t)\right]$ & 132.932 & 169.266 & 237.806 \\
\hline $\mathbb{P}\left(S^{*}=\sup _{t_{2} \leq t \leq t_{3}} S(t)\right)$ & 0.43385 & 0.33984 & 0.25305 \\
\hline $\mathbb{E}\left[S^{*} \mid S^{*}=\sup _{t_{2} \leq t \leq t_{3}} S(t)\right]$ & 146.751 & 200.064 & 305.068 \\
\hline
\end{tabular}

${ }^{5}$ The parameters used in this table are as follows: $S(0)=100, v=3 \%, t_{1}=1$, $t_{2}=2$, and $t_{3}=3$.

is positively correlated with the number of paths spending "much" time below $S(0)$ between $t_{0}$ and $t_{3}$.

These various phenomena are confirmed and even more pronounced on longer time horizons, especially in a high volatility regime, where $\left\{S^{*}=\sup _{0 \leq t \leq t_{1}} S(t)\right\}$ is by far the most likely event, as shown by Table 5 . The proportion of paths that verify $\left(S^{*}=\sup _{t_{1} \leq t \leq t_{2}} S(t)\right)$ remains noticeably stable, whatever the volatility level and the time horizon. Not surprisingly, the expected values of $S^{*}$ rise significantly on longer time horizons.

So far, the emphasis has been laid on volatility as the fundamental variable, but one might object that it is not so much $\sigma$ as the ratio $\sigma / v$ that matters. In Table 6 , the value of $v$ is significantly raised from $3 \%$ to $12 \%$. It is clear that
TABLE 6: Distribution of the global maximum over the various time intervals as a function of volatility with a larger drift coefficient than that in Tables 4 and $5^{6}$.

\begin{tabular}{llll}
\hline & $\sigma=18 \%$ & $\sigma=36 \%$ & $\sigma=64 \%$ \\
\hline $\mathbb{P}\left(S^{*}=\sup _{0 \leq t \leq t_{1}} S(t)\right)$ & 0.24639 & 0.35099 & 0.42833 \\
\hline $\mathbb{E}\left[S^{*} \mid S^{*}=\sup _{0 \leq t \leq t_{1}} S(t)\right]$ & 106.963 & 112.859 & 122.165 \\
\hline $\mathbb{P}\left(S^{*}=\sup _{t_{1} \leq t \leq t_{2}} S(t)\right)$ & 0.20214 & 0.21543 & 0.21541 \\
\hline $\mathbb{E}\left[S^{*} \mid S^{*}=\sup _{t_{1} \leq t \leq t_{2}} S(t)\right]$ & 116.995 & 132.917 & 160.696 \\
\hline $\mathbb{P}\left(S^{*}=\sup _{t_{2} \leq t \leq t_{3}} S(t)\right)$ & 0.55156 & 0.43357 & 0.35624 \\
\hline $\mathbb{E}\left[S^{*} \mid S^{*}=\sup _{t_{2} \leq t \leq t_{3}} S(t)\right]$ & 124.322 & 146.772 & 187.234 \\
\hline
\end{tabular}

${ }^{6}$ The parameters used in this table are as follows: $S(0)=100, v=12 \%, t_{1}=$ $0.25, t_{2}=0.5$, and $t_{3}=0.75$.

TABLE 7: Survival probability for various sequences of downward and upward absorbing steps as a function of volatility ${ }^{7}$.

\begin{tabular}{lccc}
\hline & $\sigma=18 \%$ & $\sigma=36 \%$ & $\sigma=64 \%$ \\
\hline$H_{1}=H_{2}=H_{3}=120$ & 0.7386128 & 0.4686822 & 0.3176924 \\
$H_{1}=H_{2}=H_{3}=80$ & 0.8617076 & 0.4973051 & 0.2497141 \\
$H_{1}=120, H_{2}=120$, & 0.7122884 & 0.1944965 & 0.0296195 \\
$H_{3}=80$ & & & \\
$H_{1}=120, H_{2}=80$, & 0.8167308 & 0.2772911 & 0.0484872 \\
$H_{3}=80$ & & & \\
$H_{1}=80, H_{2}=120$, & 0.7309655 & 0.3146737 & 0.0772276 \\
$H_{3}=120$ & & & \\
$H_{1}=80, H_{2}=80$, & 0.6983075 & 0.2255059 & 0.0425565 \\
$H_{3}=120$ & & &
\end{tabular}

${ }^{7}$ All the values reported in this table are obtained by using Proposition 1 with $\nu=3 \%, t_{1}=0.25, t_{2}=0.5, t_{3}=0.75, K_{1}=H_{1}, K_{2}=H_{2}$, and $K_{3}=H_{3}$.

the pattern observed in Tables 4 and 5 is not altered but merely shifted, with bigger absolute values for $\mathbb{P}\left(S^{*}=\right.$ $\left.\sup _{t_{2} \leq t \leq t_{3}} S(t)\right)$ relative to $\mathbb{P}\left(S^{*}=\sup _{0 \leq t \leq t_{1}} S(t)\right)$; just as in Tables 4 and 5 , the probability that $S^{*}$ is reached in the first time interval steadily increases with $\sigma$, the probability that $S^{*}$ is reached in the last time interval steadily decreases with $\sigma$, and the probability that $S^{*}$ is reached in the middle time interval is strikingly stable.

Finally, in Table 7, we take a brief look at the impact of combining both downward and upward steps in the boundary. Let us denote by $\Omega_{1}$ the set of probabilities of survival under a sequence of minima or maxima, that is, the set including $P_{[M M M]}$ and $P_{[m m m]}$, and let us denote by $\Omega_{2}$ the set of probabilities of survival under a sequence of minima and maxima, that is, the set including $P_{[M M m]}, P_{[M m m]}$, $P_{[m M M]}$, and $P_{[m m M]}$. It is noticeable that when volatility is low, the elements in $\Omega_{2}$ are never very different from those in 
$\Omega_{1}$, roughly speaking, while the value of the elements in $\Omega_{2}$ plummets versus that of the elements in $\Omega_{1}$ in a high volatility regime. The reason for the latter phenomenon is the fact that when volatility is high, the vast majority of the paths that never cross 120 until time $t_{2}$ are those that spend a lot of time in regions far beneath the starting point $S(0)=100$, so that there is little chance that these paths will always be above 80 between $t_{2}$ and $t_{3}$. Most of them will actually lie under 80 at time $t_{2}$. The same is true, to a slightly lesser extent, of the paths that never cross 120 until time $t_{1}$. Similarly, in a high volatility regime, the vast majority of the paths that never cross 80 until time $t_{2}$ or even $t_{1}$ are those that spend a lot of time in regions far above the starting point $S(0)=100$, so that there is little chance that these paths will always be below 120 between $t_{2}$ and $t_{3}$ or even between $t_{1}$ and $t_{3}$.

The moderate differences between the elements in $\Omega_{2}$ and those in $\Omega_{1}$ when volatility is low can be explained by the fact that, in such an environment, the probability of survival is high anyway, whatever the number and the location of the steps of the boundary. However, it is interesting to notice that $P_{[m m M]}$ is smaller than $P_{[M M m]}$ and $P_{[m M M]}$. Indeed, we know from Tables 2 and 3 that, in a low volatility regime, the probability of remaining above a downward boundary is higher than the probability of remaining below an upward boundary; that is, we have $P_{[m m m]}>P_{[M M M]}$. All the more so in Table 7 , since the positive drift coefficient creates an upward bias that decreases the likelihood of crossing a downward boundary. Since downward steps span two-thirds of the total time interval in $P_{[\mathrm{mm}]}$ versus one-third in both $P_{[M M m]}$ and $P_{[m M M]}$ the inequalities $P_{[m m M]}<P_{[M M m]}$ and $P_{[m m M]}<P_{[m M M]}$ show that the order in which steps with opposite direction are introduced matters more than the weight of downward steps versus upward steps; otherwise, we would have the reverse inequalities $P_{[\mathrm{mmM}]}>P_{[\mathrm{MMm}]}$ and $P_{[m m M]}>P_{[m M M]}$. In the high volatility regime, the order in which steps with opposite direction are introduced is relatively negligible for those boundaries where downward steps outweigh upward steps, that is, for $P_{[\mathrm{mmM}]}$ and $P_{[\mathrm{Mmm}]}$, while it has a considerable impact on those boundaries where upward steps outweigh downward steps, that is, for $P_{[M M m]}$ and $P_{[m M M]}$. All these properties cannot be easily "guessed" or anticipated and require an exact formula such as Proposition 1 to be established.

\section{Extension to Higher Dimension}

The kind of analytical calculations that led to Proposition 1 can be carried out in dimensions greater than three. Similar to the approach laid out in the proof of Proposition 1, for any number $n \in \mathbb{N}$ of upward steps, the survival probability of a process with dynamics defined by (1) is given by the following n-dimensional integral:

$$
\begin{aligned}
\mathbb{P} & \left(\sup _{t_{0}<t<t_{1}} X(t)<h_{1}, X\left(t_{1}\right)<k_{1}, \sup _{t_{1}<t<t_{2}} X(t)<h_{2}, X\left(t_{2}\right)\right. \\
< & \left.k_{2}, \ldots, \sup _{t_{n-1}<t<t_{n}} X(t)<h_{n}, X\left(t_{n}\right)<k_{n}\right) \\
& =\int_{D^{n}} \frac{\partial^{n}}{\partial x_{1} \partial x_{2} \cdots \partial x_{n}} N_{n}\left[\frac{x_{1}-\mu t_{1}}{\sigma \sqrt{t_{1}}}, \frac{x_{2}-\mu t_{2}}{\sigma \sqrt{t_{2}}}, \ldots,\right. \\
& \left.\frac{x_{n}-\mu t_{n}}{\sigma \sqrt{t_{n}}} ; \sqrt{\frac{t_{1}}{t_{2}}}, \ldots, \sqrt{\frac{t_{1}}{t_{n}}}, \sqrt{\frac{t_{2}}{t_{3}}}, \ldots, \sqrt{\frac{t_{2}}{t_{n}}}, \ldots, \sqrt{\frac{t_{n-1}}{t_{n}}}\right] \\
& \cdot \prod_{i=0}^{n-1}(1 \\
& \left.-\exp \left(\frac{2\left(h_{i+1}-x_{i}\right)\left(x_{i+1}-h_{i+1}\right)}{\sigma^{2}\left(t_{i+1}-t_{i}\right)}\right)\right) d x_{n} d x_{n-1} \cdots d x_{1},
\end{aligned}
$$

where the function $N_{n}\left[\left(x_{1}-\mu t_{1}\right) / \sigma \sqrt{t_{1}},\left(x_{2}-\mu t_{2}\right) / \sigma \sqrt{t_{2}}\right.$, $\ldots,\left(x_{n}-\mu t_{n}\right) / \sigma \sqrt{t_{n}} ; \sqrt{t_{1} / t_{2}}, \ldots, \sqrt{t_{1} / t_{n}}, \sqrt{t_{2} / t_{3}}, \ldots, \sqrt{t_{2} / t_{n}}$, $\left.\sqrt{t_{n-1} / t_{n}}\right]$ is the cumulative distribution function associated with the $n$-variate normal random vector $\left\langle X\left(t_{i}\right), i=1, \ldots, n\right\rangle$ and $D^{n}$ is the following product of intervals:

$$
\begin{aligned}
\left.D^{n}=\right] & \left.\left.\left.-\infty, \inf \left[k_{1}, h_{2}\right]\right] \times\right]-\infty, \inf \left[k_{2}, h_{3}\right]\right] \times \cdots \\
& \left.\times]-\infty, \inf \left[k_{n-1}, h_{n}\right]\right] .
\end{aligned}
$$

The question is, can this integral be solved as a linear combination of functions that are computable with adequate accuracy and efficiency? Although the analytical side of the problem is not trivial, the main issue is numerical. Indeed, an explicit solution involves a combination of a number $2^{n}$ of $n$-dimensional Gaussian integrals. Currently, there is no algorithm capable of evaluating the multivariate normal cumulative distribution function efficiently and with arbitrary precision as soon as the dimension of the normal integral is greater than or equal to 4 . However, it can be noticed that, as a consequence of the Markov property of the process $\{X(t), t \geq 0\}$, the joint density function associated with the $n$-variate normal random vector $\left\langle X\left(t_{i}\right), i=1, \ldots, n\right\rangle$ simplifies to

$$
\begin{aligned}
& f_{X\left(t_{1}\right), \ldots, X\left(t_{n}\right)}\left(x_{1}, \ldots, x_{n}\right) \\
& =\frac{\exp \left(-(1 / 2)\left(\left(x_{1}-\mu t_{1}\right) / \sigma \sqrt{t_{1}}\right)^{2}-\sum_{i=1}^{n-1}\left(\left(\left(\left(x_{i+1}-\mu t_{i+1}\right) / \sigma \sqrt{t_{i+1}}\right)-\sqrt{t_{i} / t_{i+1}}\left(\left(x_{i}-\mu t_{i}\right) / \sigma \sqrt{t_{i}}\right)\right)^{2} / 2\left(1-t_{i} / t_{i+1}\right)\right)\right)}{\sigma^{n}(2 \pi)^{n / 2} \prod_{i=1}^{n} \sqrt{t_{i}\left(1-t_{i-1} / t_{i}\right)}}
\end{aligned}
$$


which is simpler than the standard $n$-dimensional normal density function for computational purposes.

The integral to be solved becomes

$$
\begin{aligned}
& \int_{D^{n}} f_{X\left(t_{1}\right), \ldots, X\left(t_{n}\right)}\left(x_{1}, \ldots, x_{n}\right) \prod_{i=0}^{n-1}(1 \\
& \left.\quad-\exp \left(\frac{2\left(h_{i+1}-x_{i}\right)\left(x_{i+1}-h_{i+1}\right)}{\sigma^{2}\left(t_{i+1}-t_{i}\right)}\right)\right) d x_{n} d x_{n-1} \cdots d x_{1} .
\end{aligned}
$$

This new representation makes calculations easier both from an analytical perspective and from a numerical perspective. A general solution for any $n \in \mathbb{N}$ remains intractable, but it becomes possible to achieve a closed form solution for moderate $n$ without too much effort, although the resulting formulae will be rather bulky. In particular, for $n=5$, one can obtain the following proposition.

Proposition 2. Given $h_{1}>0$ and $k_{1} \leq h_{1}, k_{2} \leq h_{2}, k_{3} \leq h_{3}$, $k_{4} \leq h_{4}$, and $k_{5} \leq h_{5}$, we have

$$
\begin{aligned}
& \mathbb{P}\left(\sup _{t_{0}<t<t_{1}} X(t)<h_{1}, X\left(t_{1}\right)<k_{1}, \sup _{t_{1}<t<t_{2}} X(t)<h_{2},\right. \\
& X\left(t_{2}\right)<k_{2}, \sup _{t_{2}<t<t_{3}} X(t)<h_{3}, X\left(t_{3}\right)<k_{3}, \\
& \sup _{t_{3}<t<t_{4}} X(t)<h_{4}, X\left(t_{4}\right)<k_{4}, \sup _{t_{4}<t<t_{5}} X(t)<h_{5} \text {, } \\
& \left.X\left(t_{5}\right)<k_{5}\right)=\Phi_{5}\left[\frac{k_{1} \wedge h_{2}-\mu t_{1}}{\sigma \sqrt{t_{1}}}, \frac{k_{2} \wedge h_{3}-\mu t_{2}}{\sigma \sqrt{t_{2}}},\right. \\
& \frac{k_{3} \wedge h_{4}-\mu t_{3}}{\sigma \sqrt{t_{3}}}, \frac{k_{4} \wedge h_{5}-\mu t_{4}}{\sigma \sqrt{t_{4}}}, \frac{k_{5}-\mu t_{5}}{\sigma \sqrt{t_{5}}} ; \sqrt{\frac{t_{1}}{t_{2}}}, \sqrt{\frac{t_{2}}{t_{3}}}, \\
& \left.\sqrt{\frac{t_{3}}{t_{4}}}, \sqrt{\frac{t_{4}}{t_{5}}}\right]-\exp \left(\frac{2 \mu h_{1}}{\sigma^{2}}\right) \\
& \text {. } \Phi_{5}\left[\frac{k_{1} \wedge h_{2}-2 h_{1}-\mu t_{1}}{\sigma \sqrt{t_{1}}}, \frac{k_{2} \wedge h_{3}-2 h_{1}-\mu t_{2}}{\sigma \sqrt{t_{2}}},\right. \\
& \frac{k_{3} \wedge h_{4}-2 h_{1}-\mu t_{3}}{\sigma \sqrt{t_{3}}}, \frac{k_{4} \wedge h_{5}-2 h_{1}-\mu t_{4}}{\sigma \sqrt{t_{4}}}, \\
& \left.\frac{k_{5}-2 h_{1}-\mu t_{5}}{\sigma \sqrt{t_{5}}} ; \sqrt{\frac{t_{1}}{t_{2}}}, \sqrt{\frac{t_{2}}{t_{3}}}, \sqrt{\frac{t_{3}}{t_{4}}}, \sqrt{\frac{t_{4}}{t_{5}}}\right] \\
& -\exp \left(\frac{2 \mu h_{2}}{\sigma^{2}}\right) \Phi_{5}\left[\frac{k_{1} \wedge h_{2}+\mu t_{1}}{\sigma \sqrt{t_{1}}},\right. \\
& \frac{k_{2} \wedge h_{3}-2 h_{2}-\mu t_{2}}{\sigma \sqrt{t_{2}}}, \frac{k_{3} \wedge h_{4}-2 h_{2}-\mu t_{3}}{\sigma \sqrt{t_{3}}}, \\
& \frac{k_{4} \wedge h_{5}-2 h_{2}-\mu t_{4}}{\sigma \sqrt{t_{4}}}, \frac{k_{5}-2 h_{2}-\mu t_{5}}{\sigma \sqrt{t_{5}}} ;-\sqrt{\frac{t_{1}}{t_{2}}}, \sqrt{\frac{t_{2}}{t_{3}}},
\end{aligned}
$$

$$
\begin{aligned}
& \left.\sqrt{\frac{t_{3}}{t_{4}}}, \sqrt{\frac{t_{4}}{t_{5}}}\right]-\exp \left(\frac{2 \mu h_{3}}{\sigma^{2}}\right) \Phi_{5}\left[\frac{k_{1} \wedge h_{2}+\mu t_{1}}{\sigma \sqrt{t_{1}}},\right. \\
& \frac{k_{2} \wedge h_{3}+\mu t_{2}}{\sigma \sqrt{t_{2}}}, \frac{k_{3} \wedge h_{4}-2 h_{3}-\mu t_{3}}{\sigma \sqrt{t_{3}}} \text {, } \\
& \frac{k_{4} \wedge h_{5}-2 h_{3}-\mu t_{4}}{\sigma \sqrt{t_{4}}}, \frac{k_{5}-2 h_{3}-\mu t_{5}}{\sigma \sqrt{t_{5}}} ; \sqrt{\frac{t_{1}}{t_{2}}},-\sqrt{\frac{t_{2}}{t_{3}}}, \\
& \left.\sqrt{\frac{t_{3}}{t_{4}}}, \sqrt{\frac{t_{4}}{t_{5}}}\right]-\exp \left(\frac{2 \mu h_{4}}{\sigma^{2}}\right) \Phi_{5}\left[\frac{k_{1} \wedge h_{2}+\mu t_{1}}{\sigma \sqrt{t_{1}}},\right. \\
& \frac{k_{2} \wedge h_{3}+\mu t_{2}}{\sigma \sqrt{t_{2}}}, \frac{k_{3} \wedge h_{4}+\mu t_{3}}{\sigma \sqrt{t_{3}}}, \frac{k_{4} \wedge h_{5}-2 h_{4}-\mu t_{4}}{\sigma \sqrt{t_{4}}}, \\
& \left.\frac{k_{5}-2 h_{4}-\mu t_{5}}{\sigma \sqrt{t_{5}}} ; \sqrt{\frac{t_{1}}{t_{2}}}, \sqrt{\frac{t_{2}}{t_{3}}},-\sqrt{\frac{t_{3}}{t_{4}}}, \sqrt{\frac{t_{4}}{t_{5}}}\right] \\
& -\exp \left(\frac{2 \mu h_{5}}{\sigma^{2}}\right) \Phi_{5}\left[\frac{k_{1} \wedge h_{2}+\mu t_{1}}{\sigma \sqrt{t_{1}}}, \frac{k_{2} \wedge h_{3}+\mu t_{2}}{\sigma \sqrt{t_{2}}}\right. \\
& \frac{k_{3} \wedge h_{4}+\mu t_{3}}{\sigma \sqrt{t_{3}}}, \frac{k_{4} \wedge h_{5}+\mu t_{4}}{\sigma \sqrt{t_{4}}}, \frac{k_{5}-2 h_{5}-\mu t_{5}}{\sigma \sqrt{t_{5}}} ; \\
& \left.\sqrt{\frac{t_{1}}{t_{2}}}, \sqrt{\frac{t_{2}}{t_{3}}}, \sqrt{\frac{t_{3}}{t_{4}}},-\sqrt{\frac{t_{4}}{t_{5}}}\right]+\exp \left(\frac{2 \mu\left(h_{2}-h_{1}\right)}{\sigma^{2}}\right) \\
& \cdot \Phi_{5}\left[\frac{k_{1} \wedge h_{2}-2 h_{1}+\mu t_{1}}{\sigma \sqrt{t_{1}}}\right. \\
& \frac{k_{2} \wedge h_{3}-2 h_{2}+2 h_{1}-\mu t_{2}}{\sigma \sqrt{t_{2}}} \\
& \frac{k_{3} \wedge h_{4}-2 h_{2}+2 h_{1}-\mu t_{3}}{\sigma \sqrt{t_{3}}} \\
& \frac{k_{4} \wedge h_{5}-2 h_{2}+2 h_{1}-\mu t_{4}}{\sigma \sqrt{t_{4}}}, \frac{k_{5}-2 h_{2}+2 h_{1}-\mu t_{5}}{\sigma \sqrt{t_{5}}} \text {; } \\
& \left.-\sqrt{\frac{t_{1}}{t_{2}}}, \sqrt{\frac{t_{2}}{t_{3}}}, \sqrt{\frac{t_{3}}{t_{4}}}, \sqrt{\frac{t_{4}}{t_{5}}}\right]+\exp \left(\frac{2 \mu\left(h_{3}-h_{1}\right)}{\sigma^{2}}\right) \\
& \text {. } \Phi_{5}\left[\frac{k_{1} \wedge h_{2}-2 h_{1}+\mu t_{1}}{\sigma \sqrt{t_{1}}}, \frac{k_{2} \wedge h_{3}-2 h_{1}+\mu t_{2}}{\sigma \sqrt{t_{2}}},\right. \\
& \frac{k_{3} \wedge h_{4}-2 h_{3}+2 h_{1}-\mu t_{3}}{\sigma \sqrt{t_{3}}} \\
& \frac{k_{4} \wedge h_{5}-2 h_{3}+2 h_{1}-\mu t_{4}}{\sigma \sqrt{t_{4}}}, \frac{k_{5}-2 h_{3}+2 h_{1}-\mu t_{5}}{\sigma \sqrt{t_{5}}} \text {; } \\
& \left.\sqrt{\frac{t_{1}}{t_{2}}},-\sqrt{\frac{t_{2}}{t_{3}}}, \sqrt{\frac{t_{3}}{t_{4}}}, \sqrt{\frac{t_{4}}{t_{5}}}\right]+\exp \left(\frac{2 \mu\left(h_{3}-h_{2}\right)}{\sigma^{2}}\right) \\
& \text {. } \Phi_{5}\left[\frac{k_{1} \wedge h_{2}-\mu t_{1}}{\sigma \sqrt{t_{1}}}, \frac{k_{2} \wedge h_{3}-2 h_{2}+\mu t_{2}}{\sigma \sqrt{t_{2}}},\right.
\end{aligned}
$$




$$
\begin{aligned}
& \frac{k_{3} \wedge h_{4}-2 h_{3}+2 h_{2}-\mu t_{3}}{\sigma \sqrt{t_{3}}}, \\
& \frac{k_{4} \wedge h_{5}-2 h_{3}+2 h_{2}-\mu t_{4}}{\sigma \sqrt{t_{4}}}, \frac{k_{5}-2 h_{3}+2 h_{2}-\mu t_{5}}{\sigma \sqrt{t_{5}}} ; \\
& \left.-\sqrt{\frac{t_{1}}{t_{2}}},-\sqrt{\frac{t_{2}}{t_{3}}}, \sqrt{\frac{t_{3}}{t_{4}}}, \sqrt{\frac{t_{4}}{t_{5}}}\right]+\exp \left(\frac{2 \mu\left(h_{4}-h_{1}\right)}{\sigma^{2}}\right) \\
& \text {. } \Phi_{5}\left[\frac{k_{1} \wedge h_{2}-2 h_{1}+\mu t_{1}}{\sigma \sqrt{t_{1}}}, \frac{k_{2} \wedge h_{3}-2 h_{1}+\mu t_{2}}{\sigma \sqrt{t_{2}}},\right. \\
& \frac{k_{3} \wedge h_{4}-2 h_{1}+\mu t_{3}}{\sigma \sqrt{t_{3}}}, \frac{k_{4} \wedge h_{5}-2 h_{4}+2 h_{1}-\mu t_{4}}{\sigma \sqrt{t_{4}}}, \\
& \left.\frac{k_{5}-2 h_{4}+2 h_{1}-\mu t_{5}}{\sigma \sqrt{t_{5}}} ; \sqrt{\frac{t_{1}}{t_{2}}}, \sqrt{\frac{t_{2}}{t_{3}}},-\sqrt{\frac{t_{3}}{t_{4}}}, \sqrt{\frac{t_{4}}{t_{5}}}\right] \\
& +\exp \left(\frac{2 \mu\left(h_{4}-h_{2}\right)}{\sigma^{2}}\right) \Phi_{5}\left[\frac{k_{1} \wedge h_{2}-\mu t_{1}}{\sigma \sqrt{t_{1}}},\right. \\
& \frac{k_{2} \wedge h_{3}-2 h_{2}+\mu t_{2}}{\sigma \sqrt{t_{2}}}, \frac{k_{3} \wedge h_{4}-2 h_{2}+\mu t_{3}}{\sigma \sqrt{t_{3}}}, \\
& \frac{k_{4} \wedge h_{5}-2 h_{4}+2 h_{2}-\mu t_{4}}{\sigma \sqrt{t_{4}}}, \frac{k_{5}-2 h_{4}+2 h_{2}-\mu t_{5}}{\sigma \sqrt{t_{5}}} ; \\
& \left.-\sqrt{\frac{t_{1}}{t_{2}}}, \sqrt{\frac{t_{2}}{t_{3}}},-\sqrt{\frac{t_{3}}{t_{4}}}, \sqrt{\frac{t_{4}}{t_{5}}}\right]+\exp \left(\frac{2 \mu\left(h_{4}-h_{3}\right)}{\sigma^{2}}\right) \\
& \text {. } \Phi_{5}\left[\frac{k_{1} \wedge h_{2}-\mu t_{1}}{\sigma \sqrt{t_{1}}}, \frac{k_{2} \wedge h_{3}-\mu t_{2}}{\sigma \sqrt{t_{2}}},\right. \\
& \frac{k_{3} \wedge h_{4}-2 h_{3}+\mu t_{3}}{\sigma \sqrt{t_{3}}}, \frac{k_{4} \wedge h_{5}-2 h_{4}+2 h_{3}-\mu t_{4}}{\sigma \sqrt{t_{4}}}, \\
& \left.\frac{k_{5}-2 h_{4}+2 h_{3}-\mu t_{5}}{\sigma \sqrt{t_{5}}} ; \sqrt{\frac{t_{1}}{t_{2}}},-\sqrt{\frac{t_{2}}{t_{3}}},-\sqrt{\frac{t_{3}}{t_{4}}}, \sqrt{\frac{t_{4}}{t_{5}}}\right] \\
& +\exp \left(\frac{2 \mu\left(h_{5}-h_{1}\right)}{\sigma^{2}}\right) \Phi_{5}\left[\frac{k_{1} \wedge h_{2}-2 h_{1}+\mu t_{1}}{\sigma \sqrt{t_{1}}},\right. \\
& \frac{k_{2} \wedge h_{3}-2 h_{1}+\mu t_{2}}{\sigma \sqrt{t_{2}}}, \frac{k_{3} \wedge h_{4}-2 h_{1}+\mu t_{3}}{\sigma \sqrt{t_{3}}}, \\
& \frac{k_{4} \wedge h_{5}-2 h_{1}+\mu t_{4}}{\sigma \sqrt{t_{4}}}, \frac{k_{5}-2 h_{5}+2 h_{1}-\mu t_{5}}{\sigma \sqrt{t_{5}}} ; \sqrt{\frac{t_{1}}{t_{2}}}, \\
& \left.\sqrt{\frac{t_{2}}{t_{3}}}, \sqrt{\frac{t_{3}}{t_{4}}},-\sqrt{\frac{t_{4}}{t_{5}}}\right]+\exp \left(\frac{2 \mu\left(h_{5}-h_{2}\right)}{\sigma^{2}}\right) \\
& \cdot \Phi_{5}\left[\frac{k_{1} \wedge h_{2}-\mu t_{1}}{\sigma \sqrt{t_{1}}}, \frac{k_{2} \wedge h_{3}-2 h_{2}+\mu t_{2}}{\sigma \sqrt{t_{2}}},\right. \\
& \frac{k_{3} \wedge h_{4}-2 h_{2}+\mu t_{3}}{\sigma \sqrt{t_{3}}}, \frac{k_{4} \wedge h_{5}-2 h_{2}+\mu t_{4}}{\sigma \sqrt{t_{4}}}, \\
& \begin{array}{l}
\frac{k_{5}-2 h_{5}+2 h_{2}-\mu t_{5}}{\sigma \sqrt{t_{5}}} ;-\sqrt{\frac{t_{1}}{t_{2}}}, \sqrt{\frac{t_{2}}{t_{3}}}, \sqrt{\frac{t_{3}}{t_{4}}},-\sqrt{t_{4}} \\
+\exp \left(\frac{2 \mu\left(h_{5}-h_{3}\right)}{\sigma^{2}}\right) \Phi_{5}\left[\frac{k_{1} \wedge h_{2}-\mu t_{1}}{\sigma \sqrt{t_{1}}},\right.
\end{array} \\
& \frac{k_{2} \wedge h_{3}-\mu t_{2}}{\sigma \sqrt{t_{2}}}, \frac{k_{3} \wedge h_{4}-2 h_{3}+\mu t_{3}}{\sigma \sqrt{t_{3}}}, \\
& \frac{k_{4} \wedge h_{5}-2 h_{3}+\mu t_{4}}{\sigma \sqrt{t_{4}}}, \frac{k_{5}-2 h_{5}+2 h_{3}-\mu t_{5}}{\sigma \sqrt{t_{5}}} ; \sqrt{\frac{t_{1}}{t_{2}}}, \\
& \left.-\sqrt{\frac{t_{2}}{t_{3}}}, \sqrt{\frac{t_{3}}{t_{4}}},-\sqrt{\frac{t_{4}}{t_{5}}}\right]+\exp \left(\frac{2 \mu\left(h_{5}-h_{4}\right)}{\sigma^{2}}\right) \\
& \text {. } \Phi_{5}\left[\frac{k_{1} \wedge h_{2}-\mu t_{1}}{\sigma \sqrt{t_{1}}}, \frac{k_{2} \wedge h_{3}-\mu t_{2}}{\sigma \sqrt{t_{2}}}, \frac{k_{3} \wedge h_{4}-\mu t_{3}}{\sigma \sqrt{t_{3}}},\right. \\
& \frac{k_{4} \wedge h_{5}-2 h_{4}+\mu t_{4}}{\sigma \sqrt{t_{4}}}, \frac{k_{5}-2 h_{5}+2 h_{4}-\mu t_{5}}{\sigma \sqrt{t_{5}}} ; \sqrt{\frac{t_{1}}{t_{2}}}, \\
& \left.\sqrt{\frac{t_{2}}{t_{3}}},-\sqrt{\frac{t_{3}}{t_{4}}},-\sqrt{\frac{t_{4}}{t_{5}}}\right]-\exp \left(\frac{2 \mu\left(h_{3}-h_{2}+h_{1}\right)}{\sigma^{2}}\right) \\
& \text {. } \Phi_{5}\left[\frac{k_{1} \wedge h_{2}-2 h_{1}-\mu t_{1}}{\sigma \sqrt{t_{1}}},\right. \\
& \frac{k_{2} \wedge h_{3}-2 h_{2}+2 h_{1}+\mu t_{2}}{\sigma \sqrt{t_{2}}}, \\
& \frac{k_{3} \wedge h_{4}-2 h_{3}+2 h_{2}-2 h_{1}-\mu t_{3}}{\sigma \sqrt{t_{3}}}, \\
& \frac{k_{4} \wedge h_{5}-2 h_{3}+2 h_{2}-2 h_{1}-\mu t_{4}}{\sigma \sqrt{t_{4}}}, \\
& \frac{k_{5}-2 h_{3}+2 h_{2}-2 h_{1}-\mu t_{5}}{\sigma \sqrt{t_{5}}} ;-\sqrt{\frac{t_{1}}{t_{2}}},-\sqrt{\frac{t_{2}}{t_{3}}}, \sqrt{\frac{t_{3}}{t_{4}}}, \\
& \left.\sqrt{\frac{t_{4}}{t_{5}}}\right]-\exp \left(\frac{2 \mu\left(h_{4}-h_{2}+h_{1}\right)}{\sigma^{2}}\right) \\
& \text {. } \Phi_{5}\left[\frac{k_{1} \wedge h_{2}-2 h_{1}-\mu t_{1}}{\sigma \sqrt{t_{1}}},\right. \\
& \frac{k_{2} \wedge h_{3}-2 h_{2}+2 h_{1}+\mu t_{2}}{\sigma \sqrt{t_{2}}}, \\
& \frac{k_{3} \wedge h_{4}-2 h_{2}+2 h_{1}+\mu t_{3}}{\sigma \sqrt{t_{3}}}, \\
& \frac{k_{4} \wedge h_{5}-2 h_{4}+2 h_{2}-2 h_{1}-\mu t_{4}}{\sigma \sqrt{t_{4}}}, \\
& \frac{k_{5}-2 h_{4}+2 h_{2}-2 h_{1}-\mu t_{5}}{\sigma \sqrt{t_{5}}} ;-\sqrt{\frac{t_{1}}{t_{2}}}, \sqrt{\frac{t_{2}}{t_{3}}},-\sqrt{\frac{t_{3}}{t_{4}}},
\end{aligned}
$$




$$
\begin{aligned}
& \left.\sqrt{\frac{t_{4}}{t_{5}}}\right]-\exp \left(\frac{2 \mu\left(h_{4}-h_{3}+h_{1}\right)}{\sigma^{2}}\right) \\
& \text {. } \Phi_{5}\left[\frac{k_{1} \wedge h_{2}-2 h_{1}-\mu t_{1}}{\sigma \sqrt{t_{1}}}, \frac{k_{2} \wedge h_{3}-2 h_{1}-\mu t_{2}}{\sigma \sqrt{t_{2}}},\right. \\
& \frac{k_{3} \wedge h_{4}-2 h_{3}+2 h_{1}+\mu t_{3}}{\sigma \sqrt{t_{3}}}, \\
& \frac{k_{4} \wedge h_{5}-2 h_{4}+2 h_{3}-2 h_{1}-\mu t_{4}}{\sigma \sqrt{t_{4}}}, \\
& \frac{k_{5}-2 h_{4}+2 h_{3}-2 h_{1}-\mu t_{5}}{\sigma \sqrt{t_{5}}} ; \sqrt{\frac{t_{1}}{t_{2}}},-\sqrt{\frac{t_{2}}{t_{3}}},-\sqrt{\frac{t_{3}}{t_{4}}}, \\
& \left.\sqrt{\frac{t_{4}}{t_{5}}}\right]-\exp \left(\frac{2 \mu\left(h_{4}-h_{3}+h_{2}\right)}{\sigma^{2}}\right) \\
& \text {. } \Phi_{5}\left[\frac{k_{1} \wedge h_{2}-\mu t_{1}}{\sigma \sqrt{t_{1}}}, \frac{k_{2} \wedge h_{3}-2 h_{2}-\mu t_{2}}{\sigma \sqrt{t_{2}}},\right. \\
& \frac{k_{3} \wedge h_{4}-2 h_{3}+2 h_{2}+\mu t_{3}}{\sigma \sqrt{t_{3}}}, \\
& \frac{k_{4} \wedge h_{5}-2 h_{4}+2 h_{3}-2 h_{2}-\mu t_{4}}{\sigma \sqrt{t_{4}}}, \\
& \frac{k_{5}-2 h_{4}+2 h_{3}-2 h_{2}-\mu t_{5}}{\sigma \sqrt{t_{5}}} ; \sqrt{\frac{t_{1}}{t_{2}}},-\sqrt{\frac{t_{2}}{t_{3}}},-\sqrt{\frac{t_{3}}{t_{4}}}, \\
& \left.\sqrt{\frac{t_{4}}{t_{5}}}\right]-\exp \left(\frac{2 \mu\left(h_{5}-h_{2}+h_{1}\right)}{\sigma^{2}}\right) \\
& \cdot \Phi_{5}\left[\frac{k_{1} \wedge h_{2}-2 h_{1}-\mu t_{1}}{\sigma \sqrt{t_{1}}},\right. \\
& \frac{k_{2} \wedge h_{3}-2 h_{2}+2 h_{1}+\mu t_{2}}{\sigma \sqrt{t_{2}}}, \\
& \frac{k_{3} \wedge h_{4}-2 h_{2}+2 h_{1}+\mu t_{3}}{\sigma \sqrt{t_{3}}}, \\
& \frac{k_{4} \wedge h_{5}-2 h_{2}+2 h_{1}+\mu t_{4}}{\sigma \sqrt{t_{4}}}, \\
& \frac{k_{5}-2 h_{5}+2 h_{2}-2 h_{1}-\mu t_{5}}{\sigma \sqrt{t_{5}}} ;-\sqrt{\frac{t_{1}}{t_{2}}}, \sqrt{\frac{t_{2}}{t_{3}}}, \sqrt{\frac{t_{3}}{t_{4}}}, \\
& \left.-\sqrt{\frac{t_{4}}{t_{5}}}\right]-\exp \left(\frac{2 \mu\left(h_{5}-h_{3}+h_{1}\right)}{\sigma^{2}}\right) \\
& \text {. } \Phi_{5}\left[\frac{k_{1} \wedge h_{2}-2 h_{1}-\mu t_{1}}{\sigma \sqrt{t_{1}}}, \frac{k_{2} \wedge h_{3}-2 h_{1}-\mu t_{2}}{\sigma \sqrt{t_{2}}},\right. \\
& \frac{k_{3} \wedge h_{4}-2 h_{3}+2 h_{1}+\mu t_{3}}{\sigma \sqrt{t_{3}}},
\end{aligned}
$$$$
\frac{k_{4} \wedge h_{5}-2 h_{3}+2 h_{1}+\mu t_{4}}{\sigma \sqrt{t_{4}}},
$$$$
\frac{k_{5}-2 h_{5}+2 h_{3}-2 h_{1}-\mu t_{5}}{\sigma \sqrt{t_{5}}} ; \sqrt{\frac{t_{1}}{t_{2}}},-\sqrt{\frac{t_{2}}{t_{3}}}, \sqrt{\frac{t_{3}}{t_{4}}},
$$$$
\left.-\sqrt{\frac{t_{4}}{t_{5}}}\right]-\exp \left(\frac{2 \mu\left(h_{5}-h_{3}+h_{2}\right)}{\sigma^{2}}\right)
$$$$
\text { . } \Phi_{5}\left[\frac{k_{1} \wedge h_{2}-\mu t_{1}}{\sigma \sqrt{t_{1}}}, \frac{k_{2} \wedge h_{3}-2 h_{2}-\mu t_{2}}{\sigma \sqrt{t_{2}}},\right.
$$$$
\frac{k_{3} \wedge h_{4}-2 h_{3}+2 h_{2}+\mu t_{3}}{\sigma \sqrt{t_{3}}},
$$$$
\frac{k_{4} \wedge h_{5}-2 h_{3}+2 h_{2}+\mu t_{4}}{\sigma \sqrt{t_{4}}},
$$$$
\frac{k_{5}-2 h_{5}+2 h_{3}-2 h_{2}-\mu t_{5}}{\sigma \sqrt{t_{5}}} ; \sqrt{\frac{t_{1}}{t_{2}}},-\sqrt{\frac{t_{2}}{t_{3}}}, \sqrt{\frac{t_{3}}{t_{4}}},
$$$$
\left.-\sqrt{\frac{t_{4}}{t_{5}}}\right]-\exp \left(\frac{2 \mu\left(h_{5}-h_{4}+h_{1}\right)}{\sigma^{2}}\right)
$$$$
\text { . } \Phi_{5}\left[\frac{k_{1} \wedge h_{2}-2 h_{1}-\mu t_{1}}{\sigma \sqrt{t_{1}}}, \frac{k_{2} \wedge h_{3}-2 h_{1}-\mu t_{2}}{\sigma \sqrt{t_{2}}},\right.
$$$$
\frac{k_{3} \wedge h_{4}-2 h_{1}-\mu t_{3}}{\sigma \sqrt{t_{3}}}, \frac{k_{4} \wedge h_{5}-2 h_{4}+2 h_{1}+\mu t_{4}}{\sigma \sqrt{t_{4}}},
$$$$
\frac{k_{5}-2 h_{5}+2 h_{4}-2 h_{1}-\mu t_{5}}{\sigma \sqrt{t_{5}}} ; \sqrt{\frac{t_{1}}{t_{2}}}, \sqrt{\frac{t_{2}}{t_{3}}},-\sqrt{\frac{t_{3}}{t_{4}}},
$$$$
\left.-\sqrt{\frac{t_{4}}{t_{5}}}\right]-\exp \left(\frac{2 \mu\left(h_{5}-h_{4}+h_{2}\right)}{\sigma^{2}}\right)
$$$$
\text { . } \Phi_{5}\left[\frac{k_{1} \wedge h_{2}-\mu t_{1}}{\sigma \sqrt{t_{1}}}, \frac{k_{2} \wedge h_{3}-2 h_{2}-\mu t_{2}}{\sigma \sqrt{t_{2}}},\right.
$$$$
\frac{k_{3} \wedge h_{4}-2 h_{2}-\mu t_{3}}{\sigma \sqrt{t_{3}}}, \frac{k_{4} \wedge h_{5}-2 h_{4}+2 h_{2}+\mu t_{4}}{\sigma \sqrt{t_{4}}},
$$$$
\frac{k_{5}-2 h_{5}+2 h_{4}-2 h_{2}-\mu t_{5}}{\sigma \sqrt{t_{5}}} ; \sqrt{\frac{t_{1}}{t_{2}}}, \sqrt{\frac{t_{2}}{t_{3}}},-\sqrt{\frac{t_{3}}{t_{4}}},
$$$$
\left.-\sqrt{\frac{t_{4}}{t_{5}}}\right]-\exp \left(\frac{2 \mu\left(h_{5}-h_{4}+h_{3}\right)}{\sigma^{2}}\right)
$$$$
\text { . } \Phi_{5}\left[\frac{k_{1} \wedge h_{2}-\mu t_{1}}{\sigma \sqrt{t_{1}}}, \frac{k_{2} \wedge h_{3}-\mu t_{2}}{\sigma \sqrt{t_{2}}},\right.
$$$$
\frac{k_{3} \wedge h_{4}-2 h_{3}-\mu t_{3}}{\sigma \sqrt{t_{3}}}, \frac{k_{4} \wedge h_{5}-2 h_{4}+2 h_{3}+\mu t_{4}}{\sigma \sqrt{t_{4}}},
$$ 


$$
\begin{aligned}
& \frac{k_{5}-2 h_{5}+2 h_{4}-2 h_{3}-\mu t_{5}}{\sigma \sqrt{t_{5}}} ; \sqrt{\frac{t_{1}}{t_{2}}}, \sqrt{\frac{t_{2}}{t_{3}}},-\sqrt{\frac{t_{3}}{t_{4}}}, \\
& \left.-\sqrt{\frac{t_{3}}{t_{4}}},-\sqrt{\frac{t_{4}}{t_{5}}}\right]+\exp \left(\frac{2 \mu\left(h_{5}-h_{4}+h_{3}-h_{1}\right)}{\sigma^{2}}\right) \\
& \left.-\sqrt{\frac{t_{4}}{t_{5}}}\right]+\exp \left(\frac{2 \mu\left(h_{4}-h_{3}+h_{2}-h_{1}\right)}{\sigma^{2}}\right) \\
& \text {. } \Phi_{5}\left[\frac{k_{1} \wedge h_{2}-2 h_{1}+\mu t_{1}}{\sigma \sqrt{t_{1}}},\right. \\
& \frac{k_{2} \wedge h_{3}-2 h_{2}+2 h_{1}-\mu t_{2}}{\sigma \sqrt{t_{2}}}, \\
& \frac{k_{3} \wedge h_{4}-2 h_{3}+2 h_{2}-2 h_{1}+\mu t_{3}}{\sigma \sqrt{t_{3}}}, \\
& \frac{k_{4} \wedge h_{5}-2 h_{4}+2 h_{3}-2 h_{2}+2 h_{1}-\mu t_{4}}{\sigma \sqrt{t_{4}}}, \\
& \frac{k_{5}-2 h_{4}+2 h_{3}-2 h_{2}+2 h_{1}-\mu t_{5}}{\sigma \sqrt{t_{5}}} ;-\sqrt{\frac{t_{1}}{t_{2}}},-\sqrt{\frac{t_{2}}{t_{3}}}, \\
& \left.-\sqrt{\frac{t_{3}}{t_{4}}}, \sqrt{\frac{t_{4}}{t_{5}}}\right]+\exp \left(\frac{2 \mu\left(h_{5}-h_{3}+h_{2}-h_{1}\right)}{\sigma^{2}}\right) \\
& \text {. } \Phi_{5}\left[\frac{k_{1} \wedge h_{2}-2 h_{1}+\mu t_{1}}{\sigma \sqrt{t_{1}}},\right. \\
& \frac{k_{2} \wedge h_{3}-2 h_{2}+2 h_{1}-\mu t_{2}}{\sigma \sqrt{t_{2}}}, \\
& \frac{k_{3} \wedge h_{4}-2 h_{3}+2 h_{2}-2 h_{1}+\mu t_{3}}{\sigma \sqrt{t_{3}}}, \\
& \frac{k_{4} \wedge h_{5}-2 h_{3}+2 h_{2}-2 h_{1}+\mu t_{4}}{\sigma \sqrt{t_{4}}}, \\
& \frac{k_{5}-2 h_{5}+2 h_{3}-2 h_{2}+2 h_{1}-\mu t_{5}}{\sigma \sqrt{t_{5}}} ;-\sqrt{\frac{t_{1}}{t_{2}}},-\sqrt{\frac{t_{2}}{t_{3}}}, \\
& \left.\sqrt{\frac{t_{3}}{t_{4}}},-\sqrt{\frac{t_{4}}{t_{5}}}\right]+\exp \left(\frac{2 \mu\left(h_{5}-h_{4}+h_{2}-h_{1}\right)}{\sigma^{2}}\right) \\
& \text {. } \Phi_{5}\left[\frac{k_{1} \wedge h_{2}-2 h_{1}+\mu t_{1}}{\sigma \sqrt{t_{1}}},\right. \\
& \frac{k_{2} \wedge h_{3}-2 h_{2}+2 h_{1}-\mu t_{2}}{\sigma \sqrt{t_{2}}}, \\
& \frac{k_{3} \wedge h_{4}-2 h_{2}+2 h_{1}-\mu t_{3}}{\sigma \sqrt{t_{3}}}, \\
& \frac{k_{4} \wedge h_{5}-2 h_{4}+2 h_{2}-2 h_{1}+\mu t_{4}}{\sigma \sqrt{t_{4}}}, \\
& \frac{k_{5}-2 h_{5}+2 h_{4}-2 h_{2}+2 h_{1}-\mu t_{5}}{\sigma \sqrt{t_{5}}} ;-\sqrt{\frac{t_{1}}{t_{2}}}, \sqrt{\frac{t_{2}}{t_{3}}}, \\
& \text {. } \Phi_{5}\left[\frac{k_{1} \wedge h_{2}-2 h_{1}+\mu t_{1}}{\sigma \sqrt{t_{1}}}, \frac{k_{2} \wedge h_{3}-2 h_{1}+\mu t_{2}}{\sigma \sqrt{t_{2}}},\right. \\
& \frac{k_{3} \wedge h_{4}-2 h_{3}+2 h_{1}-\mu t_{3}}{\sigma \sqrt{t_{3}}}, \\
& \frac{k_{4} \wedge h_{5}-2 h_{4}+2 h_{3}-2 h_{1}+\mu t_{4}}{\sigma \sqrt{t_{4}}}, \\
& \frac{k_{5}-2 h_{5}+2 h_{4}-2 h_{3}+2 h_{1}-\mu t_{5}}{\sigma \sqrt{t_{5}}} ; \sqrt{\frac{t_{1}}{t_{2}}},-\sqrt{\frac{t_{2}}{t_{3}}}, \\
& \left.-\sqrt{\frac{t_{3}}{t_{4}}},-\sqrt{\frac{t_{4}}{t_{5}}}\right]+\exp \left(\frac{2 \mu\left(h_{5}-h_{4}+h_{3}-h_{2}\right)}{\sigma^{2}}\right) \\
& \text {. } \Phi_{5}\left[\frac{k_{1} \wedge h_{2}-\mu t_{1}}{\sigma \sqrt{t_{1}}}, \frac{k_{2} \wedge h_{3}-2 h_{2}+\mu t_{2}}{\sigma \sqrt{t_{2}}},\right. \\
& \frac{k_{3} \wedge h_{4}-2 h_{3}+2 h_{2}-\mu t_{3}}{\sigma \sqrt{t_{3}}}, \\
& \frac{k_{4} \wedge h_{5}-2 h_{4}+2 h_{3}-2 h_{2}+\mu t_{4}}{\sigma \sqrt{t_{4}}}, \\
& \frac{k_{5}-2 h_{5}+2 h_{4}-2 h_{3}+2 h_{2}-\mu t_{5}}{\sigma \sqrt{t_{5}}} ;-\sqrt{\frac{t_{1}}{t_{2}}},-\sqrt{\frac{t_{2}}{t_{3}}}, \\
& \left.-\sqrt{\frac{t_{3}}{t_{4}}},-\sqrt{\frac{t_{4}}{t_{5}}}\right] \\
& -\exp \left(\frac{2 \mu\left(h_{5}-h_{4}+h_{3}-h_{2}+h_{1}\right)}{\sigma^{2}}\right) \\
& \cdot \Phi_{5}\left[\frac{k_{1} \wedge h_{2}-2 h_{1}-\mu t_{1}}{\sigma \sqrt{t_{1}}},\right. \\
& \frac{k_{2} \wedge h_{3}-2 h_{2}+2 h_{1}+\mu t_{2}}{\sigma \sqrt{t_{2}}}, \\
& \frac{k_{3} \wedge h_{4}-2 h_{3}+2 h_{2}-2 h_{1}-\mu t_{3}}{\sigma \sqrt{t_{3}}}, \\
& \frac{k_{4} \wedge h_{5}-2 h_{4}+2 h_{3}-2 h_{2}+2 h_{1}+\mu t_{4}}{\sigma \sqrt{t_{4}}}, \\
& \frac{k_{5}-2 h_{5}+2 h_{4}-2 h_{3}+2 h_{2}-2 h_{1}-\mu t_{5}}{\sigma \sqrt{t_{5}}} ;-\sqrt{\frac{t_{1}}{t_{2}}}, \\
& \left.-\sqrt{\frac{t_{2}}{t_{3}}},-\sqrt{\frac{t_{3}}{t_{4}}},-\sqrt{\frac{t_{4}}{t_{5}}}\right] .
\end{aligned}
$$


The function $\Phi_{5}[\cdot]$ in Proposition 2 is a special 5-dimensional convolution of Gaussian densities defined by

$$
\begin{aligned}
\Phi_{5} & {\left[b_{1}, \ldots, b_{5} ; \rho_{1}, \ldots, \rho_{4}\right] } \\
& =\int_{D} \frac{\exp \left(-x_{1}^{2} / 2-\sum_{i=1}^{n-1}\left(\left(x_{i+1}-\rho_{i} x_{i}\right)^{2} / 2\left(1-\rho_{i}^{2}\right)\right)\right)}{(2 \pi)^{n / 2} \prod_{i=1}^{n-1} \sqrt{1-\rho_{i}^{2}}} d x_{5} \\
& \ldots d x_{1},
\end{aligned}
$$

$$
\begin{aligned}
& \Phi_{5}\left[b_{1}, \ldots, b_{5} ; \rho_{1}, \ldots, \rho_{4}\right]=\frac{1}{\sqrt{8 \pi^{3}}} \\
& \cdot \int_{x_{2}=-\infty}^{b_{2}} \int_{x_{3}=-\infty}^{\left(b_{3}-\rho_{2} x_{2}\right) / \sqrt{1-\rho_{2}^{2}}} \int_{x_{4}=-\infty}^{\left(b_{4}-\rho_{3}\left(x_{3} \sqrt{1-\rho_{2}^{2}}+\rho_{2} x_{2}\right)\right) / \sqrt{1-\rho_{3}^{2}}} \exp \left(-\frac{x_{2}^{2}+x_{3}^{2}+x_{4}^{2}}{2}\right) N\left[\frac{b_{1}-\rho_{1} x_{2}}{\sqrt{1-\rho_{1}^{2}}}\right] N\left[\frac{b_{5}-\rho_{4}\left(x_{4} \sqrt{1-\rho_{3}^{2}}+\rho_{3}\left(x_{3} \sqrt{1-\rho_{2}^{2}}+\rho_{2} x_{2}\right)\right)}{\sqrt{1-\rho_{4}^{2}}}\right] d x_{4} d x_{3} d x_{2},
\end{aligned}
$$

In other words, the function $\Phi_{5}$ is the integrated standardized version of the function $f_{X\left(t_{1}\right), \ldots, X\left(t_{n}\right)}\left(x_{1}, \ldots, x_{n}\right)$ given by (18) in dimension 5. The numerical dimension of this integral can be reduced by a factor of two by using the following identity: where the function $N[\cdot]$ is the usual univariate standard normal cumulative distribution function.

The quality of the numerical integration scheme given in (23) was tested by applying a classical adaptive GaussLegendre quadrature, combined with a Kronrod rule to reduce the number of required iterations. These are standard numerical techniques and it is easy to find available code or built-in functions in the usual scientific computing software. When $\left|\rho_{2}\right|$ or $\left|\rho_{3}\right|$ is close to 1 , the upper bounds of the $x_{3}$ and the $x_{4}$-integrals in (23) become very "large." To avoid numerical errors, it is safe to prespecify a maximum value for all possible integral endpoints. Given the standard normal distribution of the integrands, bounding at an absolute value of 5 will entail loss of accuracy of no more than $10^{-8}$. As we did with Proposition 1, the results obtained by evaluating Proposition 2 were compared with a conditional Monte Carlo simulation scheme in which only the endpoint values of $X(t)$ in each time subinterval $\left(t_{i-1}, t_{i}\right), i \in\{1, \ldots, 5\}$, are randomly drawn at each performed simulation; if the conditions at each $t_{i}$ are met, a cumulative variable then records the product of the conditional probabilities that the boundary has not been crossed in each $\left(t_{i-1}, t_{i}\right)$ as given by (5). A set of 1,500 random lists of parameters $\sigma, h_{i}, k_{i}$, and $t_{i}$ were drawn. A minimum $10^{-5}$ level of convergence was always observed between the numerical integration of Proposition 2 and Monte Carlo simulation on condition that a sample of 25,000,000 simulations were carried out for each of the 1,500 randomly drawn lists of parameters, using the Mersenne Twister random number generator. On an ordinary personal PC, it takes approximately 0.02 second to compute function $\Phi_{5}$ by numerical integration, so that the total time required to compute Proposition 2, which involves 32 calls to function $\Phi_{5}$, is about one second. Having to perform 25,000,000 simulations, the observed average computational time required to approximate the probability given in Proposition 2 by Monte Carlo was over 20 minutes. More detailed numerical results are reported in Table 8 .

Let us now briefly measure the effect of introducing more steps in the boundary using Proposition 2. Before numerical results are presented, it must be noticed that Proposition 2 nests an exact formula for the 5-dimensional survival probability when the boundary is downward, that is, for the following probability:

$$
\begin{gathered}
\mathbb{P}\left(\inf _{t_{0}<t<t_{1}} X(t)>h_{1}, X\left(t_{1}\right)>k_{1}, \inf _{t_{1}<t<t_{2}} X(t)>h_{2}, X\left(t_{2}\right)\right. \\
>k_{2}, \inf _{t_{2}<t<t_{3}} X(t)>h_{3}, X\left(t_{3}\right)>k_{3}, \inf _{t_{3}<t<t_{4}} X(t) \\
\left.>h_{4}, X\left(t_{4}\right)>k_{4}, \inf _{t_{4}<t<t_{5}} X(t)>h_{5}, X\left(t_{5}\right)>k_{5}\right) \\
\forall h_{1}>0, k_{1} \geq h_{1}, k_{2} \geq h_{2}, k_{3} \geq h_{3}, k_{4} \geq h_{4}, k_{5} \geq h_{5} .
\end{gathered}
$$

Indeed, one can switch from the survival probability with an upward boundary to the survival probability with a downward boundary simply by multiplying each $b_{i}$ by the coefficient $(-1)$ and by taking $k_{i} \vee h_{i}$ instead of $k_{i} \wedge h_{i}$ in each $\Phi_{5}$ function in Proposition 2; $i \in\{1,2,3,4,5\}$. Both lower and upper boundaries are thus taken into consideration in Table 9, the setting of which is similar to those of Tables 2 and 3 , in order to allow for a meaningful comparison. In particular, the drift and volatility parameters are the same, and the weighted average of the steps is almost identical; but the total time $[0,0.75]$ is now divided into five subintervals of equal length instead of three. As a result, the widening and the shrinking of the upper and of the lower boundaries are a little smoother than those in Tables 2 and 3; that is, there are slightly more frequent changes in the boundaries and these changes are slightly less abrupt. When comparing Table 9 on one hand and Tables 2 and 3 on the other hand, the most noticeable fact is the fact that the sensitivity of the survival probability with respect to the number of steps in the boundary now depends more apparently on volatility: a five-step boundary is less "risky" than a three-step one when volatility is low or intermediate but it is more "risky" when volatility is high, whether the boundary is upward or downward and whether it widens or shrinks. Besides, in a low volatility regime, the survival probability is higher when the boundary widens than when it shrinks, while the reverse 
TABle 8: Numerical comparison between Proposition 2 and conditional Monte Carlo (CMC) approximation ${ }^{7}$

\begin{tabular}{lcccc}
\hline & Proposition 2 & CMC & CMC & CMC \\
& & 500,000 & $5,000,000$ & $25,000,000$ \\
\hline Average divergence (\%) & 0 & 0.57311 & 0.02378 & 0.00005 \\
Maximum divergence (\%) & 0 & 1.09239 & 0.14412 & 0.00948 \\
Computational time (seconds) & 0.81 & 30.45 & 297.43 & 1436.76 \\
\hline
\end{tabular}

${ }^{7}$ The average divergence is the average of the absolute values of the differences between Proposition 2 and CMC divided by Proposition 2 out of a sample of 1,500 lists of randomly drawn parameters.

The maximum divergence is the maximum of the absolute values of the differences between Proposition 2 and CMC divided by Proposition 2 out of a sample of 1,500 lists of randomly drawn parameters.

Computational time is measured on an i $2.5 \mathrm{Ghz}$ processor.

TABLE 9: Survival probability when the absorbing boundary includes five steps ${ }^{8}$.

\begin{tabular}{lcc}
\hline & $\sigma=18 \%$ & $\sigma=36 \%$ \\
\hline$t_{1}=0.15, t_{2}=0.3, t_{3}=0.45, t_{4}=0.6, t_{5}=0.75$ & 0.7797497 & 0.5520988 \\
$H_{1}=132, H_{2}=130, H_{3}=128, H_{4}=124, H_{5}=120$ & 0.8931785 & 0.5632040 \\
\hline$t_{1}=0.15, t_{2}=0.3, t_{3}=0.45, t_{4}=0.6, t_{5}=0.75$ & 0.8223448 & 0.3975464 \\
$H_{1}=120, H_{2}=124, H_{3}=128, H_{4}=130, H_{5}=132$ & 0.5312413 \\
\hline$t_{1}=0.15, t_{2}=0.3, t_{3}=0.45, t_{4}=0.6, t_{5}=0.75$ & 0.9677121 & 0.6234348 \\
$H_{1}=68, H_{2}=70, H_{3}=72, H_{4}=76, H_{5}=80$ & 0.3060088 \\
\hline$t_{1}=0.15, t_{2}=0.3, t_{3}=0.45, t_{4}=0.6, t_{5}=0.75$ & 0.2883722 \\
$H_{1}=80, H_{2}=76, H_{3}=72, H_{4}=70, H_{5}=68$ & \\
${ }^{8}$ All the values reported in this table are obtained by using Proposition 2 with $S(0)=100, v=3 \%, K_{1}=H_{1}, K_{2}=H_{2}, K_{3}=H_{3}, K_{4}=H_{4}$, and $K_{5}=H_{5}$.
\end{tabular}

${ }^{8}$ All the values reported in this table are obtained by using Proposition 2 with $S(0)=100, v=3 \%, K_{1}=H_{1}, K_{2}=H_{2}, K_{3}=H_{3}, K_{4}=H_{4}$, and $K_{5}=H_{5}$.

TABLE 10: Distribution of the global maximum over the various time intervals as a function of volatility using Proposition $2^{9}$.

\begin{tabular}{llll}
\hline & $\sigma=18 \%$ & $\sigma=36 \%$ & $\sigma=64 \%$ \\
\hline $\mathbb{P}\left(S^{*}=\sup _{0 \leq t \leq t_{1}} S(t)\right)$ & 0.27746 & 0.31857 & 0.36216 \\
\hline $\mathbb{P}\left(S^{*}=\sup _{t_{1} \leq t \leq t_{2}} S(t)\right)$ & 0.13694 & 0.14482 & 0.15128 \\
\hline $\mathbb{P}\left(S^{*}=\sup _{t_{2} \leq t \leq t_{3}} S(t)\right)$ & 0.12837 & 0.12831 & 0.12654 \\
\hline $\mathbb{P}\left(S^{*}=\sup _{t_{3} \leq t \leq t_{4}} S(t)\right)$ & 0.14396 & 0.13584 & 0.12672 \\
\hline $\mathbb{P}\left(S^{*}=\sup _{t_{4} \leq t \leq t_{5}} S(t)\right)$ & 0.31325 & 0.27243 & 0.23327 \\
\hline
\end{tabular}

${ }^{9}$ The parameters used in this table are as follows: $S(0)=100, v=3 \%, t_{1}=$ $0.15, t_{2}=0.3, t_{3}=0.45, t_{4}=0.6$, and $t_{5}=0.75$.

holds in a high volatility regime, whether the boundary is upward or downward. That observation is in line with the findings derived from Tables 2 and 3.

Finally, in Table 10, we compute the probabilities that the global maximum of $S(t)$ over $\left[t_{0}, t_{5}\right]$, denoted by $S^{*}$, lies within $\left(t_{0}, t_{1}\right)$ or $\left(t_{1}, t_{2}\right)$ or $\left(t_{2}, t_{3}\right)$ or $\left(t_{3}, t_{4}\right)$ or $\left(t_{4}, t_{5}\right)$, as we did in Table 4 when the total time interval was divided in three. The parameters are the same as in Table 4 . The results in Table 10 are very similar to those in Table 4 . When volatility is low, there is a concentration of probability mass at the end of the process lifetime and, to a slightly lesser extent, at its beginning. When volatility is high, the probability mass is located mostly at the beginning but remains significant at the end. The middle region is always the least likely outcome and its probability mass is strikingly stable, whatever the volatility level.

\section{Extension to Two-Sided Boundaries}

The method developed in this paper can also be applied to two-sided boundaries. This final section shows how to compute the survival probability when dealing with a twosided two-step boundary. All assumptions and notations are identical as in the previous sections, except that the boundary now consists in the combination of an upper line segment $h_{1}$ and a lower line segment $h_{2}$ during time interval $\left[0, t_{1}\right]$ and an upper line segment $h_{3}$ and a lower line segment $h_{4}$ during time interval $\left[t_{1}, t_{2}\right]$. In other words, the diffusion process $\{X(t), t \geq 0\}$ defined in (1) may be absorbed either from above or from below in each of the two successive time intervals. The following proposition provides a closed form formula for the survival probability in this particular case.

Proposition 3. Let $\{X(t), t \geq 0\}$ be an arithmetic Brownian motion under a probability measure $\mathbb{P}$, as defined in Section 1 , and let

(i) $h_{1}, h_{2}, h_{3}$, and $h_{4}$ be four real constants such that $h_{1}>$ $0, h_{2}<0$, and $h_{3}>h_{4}$,

(ii) $k_{1}$ and $k_{2}$ be two real constants such that $k_{1}>h_{2}$ and $k_{2}>h_{4}$,

(iii) $u_{1}=\min \left(h_{1}, k_{1}, h_{3}\right), u_{2}=\max \left(h_{2}, h_{4}\right)$, and $u_{3}=$ $\min \left(h_{3}, k_{2}\right)$,

(iv) $t_{1}$ and $t_{2}$ be two nonrandom times such that $t_{2} \geq t_{1} \geq 0$. 
Then,

$$
\begin{aligned}
& \mathbb{P}\left(\sup _{0 \leq t \leq t_{1}} X(t) \leq h_{1}, \inf _{0 \leq t \leq t_{1}} X(t) \geq h_{2}, X\left(t_{1}\right) \leq k_{1}, \sup _{t_{1} \leq t \leq t_{2}} X(t) \leq h_{3}, \inf _{t_{1} \leq t \leq t_{2}} X(t) \geq h_{4}, X\left(t_{2}\right) \leq k_{2}\right) \\
& =\sum_{m=-\infty}^{\infty} \sum_{n=-\infty}^{\infty} \exp \left(\frac{2 \mu}{\sigma^{2}}\left(m\left(h_{1}-h_{2}\right)+n\left(h_{3}-h_{4}\right)\right)\right) \\
& \left\{N_{2}\left[\frac{u_{1}-2 m\left(h_{1}-h_{2}\right)-\mu t_{1}}{\sigma \sqrt{t_{1}}}, \frac{u_{3}-2 n\left(h_{3}-h_{4}\right)-2 m\left(h_{1}-h_{2}\right)-\mu t_{2}}{\sigma \sqrt{t_{2}}} ; \sqrt{\frac{t_{1}}{t_{2}}}\right]\right. \\
& -N_{2}\left[\frac{u_{2}-2 m\left(h_{1}-h_{2}\right)-\mu t_{1}}{\sigma \sqrt{t_{1}}}, \frac{u_{3}-2 n\left(h_{3}-h_{4}\right)-2 m\left(h_{1}-h_{2}\right)-\mu t_{2}}{\sigma \sqrt{t_{2}}} ; \sqrt{\frac{t_{1}}{t_{2}}}\right] \\
& -N_{2}\left[\frac{u_{1}-2 m\left(h_{1}-h_{2}\right)-\mu t_{1}}{\sigma \sqrt{t_{1}}}, \frac{h_{4}-2 n\left(h_{3}-h_{4}\right)-2 m\left(h_{1}-h_{2}\right)-\mu t_{2}}{\sigma \sqrt{t_{2}}} ; \sqrt{\frac{t_{1}}{t_{2}}}\right] \\
& \left.+N_{2}\left[\frac{u_{2}-2 m\left(h_{1}-h_{2}\right)-\mu t_{1}}{\sigma \sqrt{t_{1}}}, \frac{h_{4}-2 n\left(h_{3}-h_{4}\right)-2 m\left(h_{1}-h_{2}\right)-\mu t_{2}}{\sigma \sqrt{t_{2}}} ; \sqrt{\frac{t_{1}}{t_{2}}}\right]\right\} \\
& -\sum_{m=-\infty}^{\infty} \sum_{n=-\infty}^{\infty} \exp \left(\frac{2 \mu}{\sigma^{2}}\left[h_{2}-m\left(h_{1}-h_{2}\right)+n\left(h_{3}-h_{4}\right)\right]\right) \\
& \left\{N_{2}\left[\frac{u_{1}-2 h_{2}+2 m\left(h_{1}-h_{2}\right)-\mu t_{1}}{\sigma \sqrt{t_{1}}}, \frac{u_{3}-2 h_{2}-2 n\left(h_{3}-h_{4}\right)+2 m\left(h_{1}-h_{2}\right)-\mu t_{2}}{\sigma \sqrt{t_{2}}} ; \sqrt{\frac{t_{1}}{t_{2}}}\right]\right. \\
& -N_{2}\left[\frac{u_{2}-2 h_{2}+2 m\left(h_{1}-h_{2}\right)-\mu t_{1}}{\sigma \sqrt{t_{1}}}, \frac{u_{3}-2 h_{2}-2 n\left(h_{3}-h_{4}\right)+2 m\left(h_{1}-h_{2}\right)-\mu t_{2}}{\sigma \sqrt{t_{2}}} ; \sqrt{\frac{t_{1}}{t_{2}}}\right] \\
& -N_{2}\left[\frac{u_{1}-2 h_{2}+2 m\left(h_{1}-h_{2}\right)-\mu t_{1}}{\sigma \sqrt{t_{1}}}, \frac{h_{4}-2 h_{2}-2 n\left(h_{3}-h_{4}\right)+2 m\left(h_{1}-h_{2}\right)-\mu t_{2}}{\sigma \sqrt{t_{2}}} ; \sqrt{\frac{t_{1}}{t_{2}}}\right] \\
& \left.+N_{2}\left[\frac{u_{2}-2 h_{2}+2 m\left(h_{1}-h_{2}\right)-\mu t_{1}}{\sigma \sqrt{t_{1}}}, \frac{h_{4}-2 h_{2}-2 n\left(h_{3}-h_{4}\right)+2 m\left(h_{1}-h_{2}\right)-\mu t_{2}}{\sigma \sqrt{t_{2}}} ; \sqrt{\frac{t_{1}}{t_{2}}}\right]\right\} \\
& -\sum_{m=-\infty}^{\infty} \sum_{n=-\infty}^{\infty} \exp \left(\frac{2 \mu}{\sigma^{2}}\left[h_{4}-m\left(h_{1}-h_{2}\right)-n\left(h_{3}-h_{4}\right)\right]\right) \\
& \left\{N_{2}\left[\frac{u_{1}-2 m\left(h_{1}-h_{2}\right)+\mu t_{1}}{\sigma \sqrt{t_{1}}}, \frac{u_{3}-2 h_{4}+2 n\left(h_{3}-h_{4}\right)+2 m\left(h_{1}-h_{2}\right)-\mu t_{2}}{\sigma \sqrt{t_{2}}} ;-\sqrt{\frac{t_{1}}{t_{2}}}\right]\right. \\
& -N_{2}\left[\frac{u_{2}-2 m\left(h_{1}-h_{2}\right)+\mu t_{1}}{\sigma \sqrt{t_{1}}}, \frac{u_{3}-2 h_{4}+2 n\left(h_{3}-h_{4}\right)+2 m\left(h_{1}-h_{2}\right)-\mu t_{2}}{\sigma \sqrt{t_{2}}} ;-\sqrt{\frac{t_{1}}{t_{2}}}\right] \\
& -N_{2}\left[\frac{u_{1}-2 m\left(h_{1}-h_{2}\right)+\mu t_{1}}{\sigma \sqrt{t_{1}}}, \frac{-h_{4}+2 n\left(h_{3}-h_{4}\right)+2 m\left(h_{1}-h_{2}\right)-\mu t_{2}}{\sigma \sqrt{t_{2}}} ;-\sqrt{\frac{t_{1}}{t_{2}}}\right] \\
& \left.+N_{2}\left[\frac{u_{2}-2 m\left(h_{1}-h_{2}\right)+\mu t_{1}}{\sigma \sqrt{t_{1}}}, \frac{-h_{4}+2 n\left(h_{3}-h_{4}\right)+2 m\left(h_{1}-h_{2}\right)-\mu t_{2}}{\sigma \sqrt{t_{2}}} ;-\sqrt{\frac{t_{1}}{t_{2}}}\right]\right\} \\
& +\sum_{m=-\infty}^{\infty} \sum_{n=-\infty}^{\infty} \exp \left(\frac{2 \mu}{\sigma^{2}}\left[h_{4}-h_{2}+m\left(h_{1}-h_{2}\right)-n\left(h_{3}-h_{4}\right)\right]\right)
\end{aligned}
$$




$$
\begin{aligned}
& \cdot N_{2}\left[\frac{u_{1}-2 h_{2}+2 m\left(h_{1}-h_{2}\right)+\mu t_{1}}{\sigma \sqrt{t_{1}}}, \frac{u_{3}-2 h_{4}+2 h_{2}+2 n\left(h_{3}-h_{4}\right)-2 m\left(h_{1}-h_{2}\right)-\mu t_{2}}{\sigma \sqrt{t_{2}}} ;-\sqrt{\frac{t_{1}}{t_{2}}}\right] \\
& -N_{2}\left[\frac{u_{2}-2 h_{2}+2 m\left(h_{1}-h_{2}\right)+\mu t_{1}}{\sigma \sqrt{t_{1}}}, \frac{u_{3}-2 h_{4}+2 h_{2}+2 n\left(h_{3}-h_{4}\right)-2 m\left(h_{1}-h_{2}\right)-\mu t_{2}}{\sigma \sqrt{t_{2}}} ;-\sqrt{\frac{t_{1}}{t_{2}}}\right] \\
& -N_{2}\left[\frac{u_{1}-2 h_{2}+2 m\left(h_{1}-h_{2}\right)+\mu t_{1}}{\sigma \sqrt{t_{1}}}, \frac{-h_{4}+2 h_{2}+2 n\left(h_{3}-h_{4}\right)-2 m\left(h_{1}-h_{2}\right)-\mu t_{2}}{\sigma \sqrt{t_{2}}} ;-\sqrt{\frac{t_{1}}{t_{2}}}\right] \\
& \left.+N_{2}\left[\frac{u_{2}-2 h_{2}+2 m\left(h_{1}-h_{2}\right)+\mu t_{1}}{\sigma \sqrt{t_{1}}}, \frac{-h_{4}+2 h_{2}+2 n\left(h_{3}-h_{4}\right)-2 m\left(h_{1}-h_{2}\right)-\mu t_{2}}{\sigma \sqrt{t_{2}}} ;-\sqrt{\frac{t_{1}}{t_{2}}}\right]\right\},
\end{aligned}
$$

where $N_{2}[\cdot, \cdot ; \rho]$ is the bivariate standard normal cumulative distribution function with correlation coefficient $\rho$, which can be numerically evaluated with double precision and in less than 1/100 second using the algorithm by Genz [17]. Notice that an elementary modification of Proposition 3 provides the corresponding survival probability for a geometric Brownian motion similar to the case of one-sided boundaries in Section 3. Indeed, if $\{X(t), t \geq 0\}$ is driven by

$$
d X(t)=\mu X(t) d t+\sigma X(t) d B(t)
$$

under the probability measure $\mathbb{P}$, then, to obtain the survival probability

$$
\begin{aligned}
& \mathbb{P}\left(\sup _{0 \leq t \leq t_{1}} X(t) \leq H_{1}, \inf _{0 \leq t \leq t_{1}} X(t) \geq H_{2}, X\left(t_{1}\right)\right. \\
& \quad \leq K_{1}, \sup _{t_{1} \leq t \leq t_{2}} X(t) \leq H_{3}, \inf _{t_{1} \leq t \leq t_{2}} X(t) \geq H_{4}, X\left(t_{2}\right) \\
& \left.\quad \leq K_{2}\right)
\end{aligned}
$$

where $H_{1}, H_{2}, H_{3}, H_{4}, K_{1}$, and $K_{2}$ are all positive and $H_{1}>$ $X(0), H_{2}<X(0), H_{3}>H_{4}, K_{1}>H_{2}$, and $K_{2}>H_{4}$, it suffices to apply the following substitutions in Proposition 3:

$$
\begin{aligned}
h_{i} & =\ln \left(\frac{H_{i}}{X(0)}\right), \\
k_{i} & =\ln \left(\frac{K_{i}}{X(0)}\right), \\
\mu & =\mu-\frac{\sigma^{2}}{2}
\end{aligned}
$$

as shown by an elementary application of Ito's lemma to $\ln (X(t) / X(0))$.

From a numerical standpoint, the double infinite series can be truncated in a simple manner by setting a convergence threshold such that no further terms are added once the difference between two successive finite sums of the double series becomes smaller than that prespecified level. A small number of terms in the infinite series will suffice to achieve adequate convergence for most practical purposes, so that the computational time implied by Proposition 3 will typically be around one second on an ordinary personal computer.

Proof of Proposition 3. As a detailed proof of Proposition 3 is quite cumbersome, only the structure of the proof is given here; a detailed proof is available from the author upon request.

By conditioning on $X\left(t_{1}\right)$ and $X\left(t_{2}\right)$ and by using the weak Markov property of the process $\{X(t), t \geq 0\}$, the sought probability, denoted by $p$, can be written as the solution of the following integration problem:

$$
p=\int_{u_{2}}^{u_{1}} \int_{h_{4}}^{u_{3}} f_{1}\left(x_{1}, x_{2}\right) f_{2}\left(x_{1}\right) f_{3}\left(x_{1}, x_{2}\right) d x_{2} d x_{1},
$$

where

$$
\begin{aligned}
& f_{1}\left(x_{1}, x_{2}\right)=\mathbb{P}\left(X\left(t_{1}\right) \in d x_{1}, X\left(t_{2}\right) \in d x_{2}\right) \\
& f_{2}\left(x_{1}\right)=\mathbb{P}\left(\sup _{0 \leq t \leq t_{1}} X(t) \leq h_{1}, \inf _{0 \leq t \leq t_{1}} X(t) \geq h_{2} \mid X\left(t_{1}\right)\right. \\
& \left.\quad \in d x_{1}\right), \\
& f_{3}\left(x_{1}, x_{2}\right)=\mathbb{P}\left(\sup _{t_{1} \leq t \leq t_{2}} X(t) \leq h_{3}, \inf _{t_{1} \leq t \leq t_{2}} X(t)\right. \\
& \left.\quad \geq h_{4} \mid X\left(t_{1}\right) \in d x_{1}, X\left(t_{2}\right) \in d x_{2}\right) .
\end{aligned}
$$

The pair $\left(X\left(t_{1}\right), X\left(t_{2}\right)\right)$ follows a bivariate normal distribution, so the function $f_{1}\left(x_{1}, x_{2}\right)$ is known. lemma. 
Lemma 4. Let $q$ be the conditional probability defined by

$$
\begin{aligned}
q & =\mathbb{P}\left(\sup _{t_{i} \leq t \leq t_{j}} X(t) \leq b, \inf _{t_{i} \leq t \leq t_{j}} X(t) \geq a \mid X\left(t_{i}\right)\right. \\
& \left.\in d x_{i}, X\left(t_{j}\right) \in d x_{j}\right)
\end{aligned}
$$

where $x_{i}, x_{j}, a$, and $b$ are real constants such that $b>a, b \geq$ $x_{i}>a$, and $b \geq x_{j}>a$, and $t_{i}$ and $t_{j}$ are two nonrandom times such that $t_{j} \geq t_{i}>0$.

Then,

$$
\begin{aligned}
q & =\sum_{n=-\infty}^{\infty} \exp \left(\frac{2 n(b-a)\left(x_{j}-x_{i}-n(b-a)\right)}{\sigma^{2}\left(t_{j}-t_{i}\right)}\right) \\
& -\sum_{n=-\infty}^{\infty} \exp \left(\frac{2\left(b-x_{i}-n(b-a)\right)\left(x_{j}-b+n(b-a)\right)}{\sigma^{2}\left(t_{j}-t_{i}\right)}\right) .
\end{aligned}
$$

To provide an outline of proof of Lemma 4, let us consider the following cumulative distribution function, denoted by $\varphi$ :

$$
\begin{aligned}
\varphi & =\mathbb{P}\left(X\left(t_{i}\right) \leq x_{i}, \sup _{t_{i} \leq t \leq t_{j}} X(t) \leq b, \inf _{t_{i} \leq t \leq t_{j}} X(t)\right. \\
& \left.\geq a, X\left(t_{j}\right) \leq x_{j}\right) .
\end{aligned}
$$

$\varphi$ can be expressed as the solution of the following integration problem:

$$
\varphi=\int_{a}^{x_{i}} g_{1}(y) g_{2}(y) d y
$$

where

$$
\begin{aligned}
& g_{1}(y)=\mathbb{P}\left(X\left(t_{i}\right) \in d y\right) \\
& g_{2}(y)=\mathbb{P}\left(\sup _{t_{i} \leq t \leq t_{j}} X(t) \leq b, \inf _{t_{i} \leq t \leq t_{j}} X(t) \geq a, X\left(t_{j}\right)\right. \\
& \left.\quad \leq x_{j} \mid X\left(t_{i}\right) \in d y\right) .
\end{aligned}
$$

The function $g_{1}(y)$ is just a normal density function. By integrating the classical formula for the joint distribution of the maximum, the minimum, and the endpoint of a Brownian motion on a closed time interval (Cox and Miller [19]), it can be shown that the function $g_{2}(y)$ is equal to

$$
\begin{aligned}
& g_{2}(y)=\sum_{n=-\infty}^{\infty} \exp \left(\frac{2 n \mu(b-a)}{\sigma^{2}}\right) \\
& \cdot\left[N\left(\frac{x_{j}-y-\mu\left(t_{j}-t_{i}\right)-2 n(b-a)}{\sigma \sqrt{t_{j}-t_{i}}}\right)\right.
\end{aligned}
$$

$$
\begin{aligned}
& \left.-N\left(\frac{a-y-\mu\left(t_{j}-t_{i}\right)-2 n(b-a)}{\sigma \sqrt{t_{j}-t_{i}}}\right)\right] \\
& -\sum_{n=-\infty}^{\infty} \exp \left(\frac{2 \mu(a-y-n(b-a))}{\sigma^{2}}\right) \\
& \cdot N\left(\frac{x_{j}-2 a+y-\mu\left(t_{j}-t_{i}\right)+2 n(b-a)}{\sigma \sqrt{t_{j}-t_{i}}}\right) \\
& \left.-N\left(\frac{-a+y-\mu\left(t_{j}-t_{i}\right)+2 n(b-a)}{\sigma \sqrt{t_{j}-t_{i}}}\right)\right],
\end{aligned}
$$

where $N[\cdot]$ is the univariate standard normal cumulative distribution function.

Plugging $g_{1}(y)$ and $g_{2}(y)$ in (34), $\varphi$ can be obtained in closed form as an infinite series of a linear combination of eight bivariate standard normal cumulative distribution functions. Differentiating $\varphi$ with respect to $x_{i}$ and then with respect to $x_{j}$ and dividing the resulting quantity by $\mathbb{P}\left(X\left(t_{i}\right) \in\right.$ $\left.d x_{i}, X\left(t_{j}\right) \in d x_{j}\right)$ yield Lemma 4 .

Once endowed with Lemma 4 , the functions $f_{2}\left(x_{1}\right)$ and $f_{3}\left(x_{1}, x_{2}\right)$ can be plugged in (29). The rest of the proof consists in performing the necessary calculations to solve (29) in closed form and thus obtain Proposition 3.

\section{Conclusion}

In this paper, closed form solutions are obtained for the survival probability of an arithmetic or a geometric Brownian motion when the boundary is a step function. Although only up to five steps are considered, it is a straightforward extension to write down formulae when the boundary includes more than five steps. However, the obtained results are too cumbersome to be printed in Journal format. Numerical testing was carried out and showed that it was still possible to achieve an excellent level of accuracy and efficiency, for all practical purposes, when the required numerical integration is performed in dimension seven. In "much" higher dimensions, the numerical integration required by analytical formulae such as the ones provided in this paper may not be competitive with regard to a conditional Monte Carlo simulation scheme, as the efficiency of the latter decreases only linearly with dimension. Nonetheless, exact results obtained with a formula for a smaller number of steps will still be very useful as control variates allowing for a substantial variance reduction of the Monte Carlo approximation.

\section{Conflict of Interests}

The author declares that there is no conflict of interests regarding the publication of this paper. 


\section{References}

[1] L. Wang and K. Pötzelberger, "Crossing probabilities for diffusion processes with piecewise continuous boundaries," Methodology and Computing in Applied Probability, vol. 9, no. 1, pp. 2140, 2007.

[2] L. Breiman, "First exit times from a square root boundary," in Proceedings of the 5th Berkeley Symposium on Mathematical Statistics and Probability, vol. 2, pp. 9-16, 1966.

[3] S. Sato, "Evaluation of the first-passage time probability to a square root boundary for the Wiener process," Journal of Applied Probability, vol. 14, no. 4, pp. 850-856, 1977.

[4] P. Groeneboom, "Brownian motion with a parabolic drift and Airy functions," Probability Theory and Related Fields, vol. 81, no. 1, pp. 79-109, 1989.

[5] H. E. Daniels, "Approximating the first crossing-time density for a curved boundary," Bernoulli, vol. 2, no. 2, pp. 133-143, 1996.

[6] C. Park and F. J. Schuurmann, "Evaluations of barrier-crossing probabilities of Wiener paths," Journal of Applied Probability, vol. 13, no. 2, pp. 267-275, 1976.

[7] C. Jennen and H. R. Lerche, "First exit densities of Brownian motion through one-sided moving boundaries," Zeitschrift für Wahrscheinlichkeitstheorie und Verwandte Gebiete, vol. 55, no. 2, pp. 133-148, 1981.

[8] P. Salminen, "On the first hitting time and the last exit time for a Brownian motion to/from a moving boundary," Advances in Applied Probability, vol. 20, no. 2, pp. 411-426, 1988.

[9] T. H. Scheike, "A boundary-crossing result for Brownian motion," Journal of Applied Probability, vol. 29, no. 2, pp. 448453, 1992.

[10] A. Novikov, V. Frishling, and N. Kordzakhia, "Approximations of boundary crossing probabilities for a Brownian motion," Journal of Applied Probability, vol. 36, no. 4, pp. 1019-1030, 1999.

[11] J. C. Fu and T.-L. Wu, "Linear and nonlinear boundary crossing probabilities for Brownian motion and related processes," Journal of Applied Probability, vol. 47, no. 4, pp. 1058-1071, 2010.

[12] L. Alili, P. Patie, and J. L. Pedersen, "Representations of the first hitting time density of an Ornstein-Uhlenbeck process," Stochastic Models, vol. 21, no. 4, pp. 967-980, 2005.

[13] C. F. Lo and C. H. Hui, "Computing the first passage time density of a time-dependent Ornstein-Uhlenbeck process to a moving boundary," Applied Mathematics Letters, vol. 19, no. 12, pp. 1399-1405, 2006.

[14] J. Durbin, "The first-passage density of a continuous Gaussian process to a general boundary," Journal of Applied Probability, vol. 22, no. 1, pp. 99-122, 1985.

[15] K. Pötzelberger and L. Wang, "Boundary crossing probability for Brownian motion," Journal of Applied Probability, vol. 38, no. 1, pp. 152-164, 2001.

[16] A. N. Downes and K. Borovkov, "First passage densities and boundary crossing probabilities for diffusion processes," Methodology and Computing in Applied Probability, vol. 10, no. 4, pp. 621-644, 2008.

[17] A. Genz, "Numerical computation of rectangular bivariate and trivariate normal and $t$ probabilities," Statistics and Computing, vol. 14, no. 3, pp. 251-260, 2004.

[18] J. S. Dagpunar, Simulation and Monte Carlo, John Wiley \& Sons, 2007.

[19] D. R. Cox and H. D. Miller, The Theory of Stochastic Processes, Methuen, London, UK, 1965. 


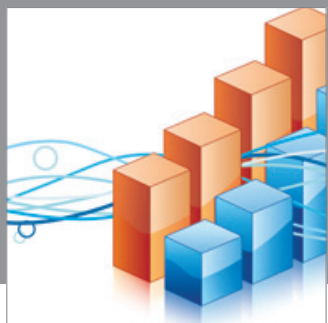

Advances in

Operations Research

mansans

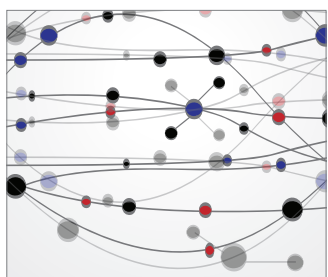

The Scientific World Journal
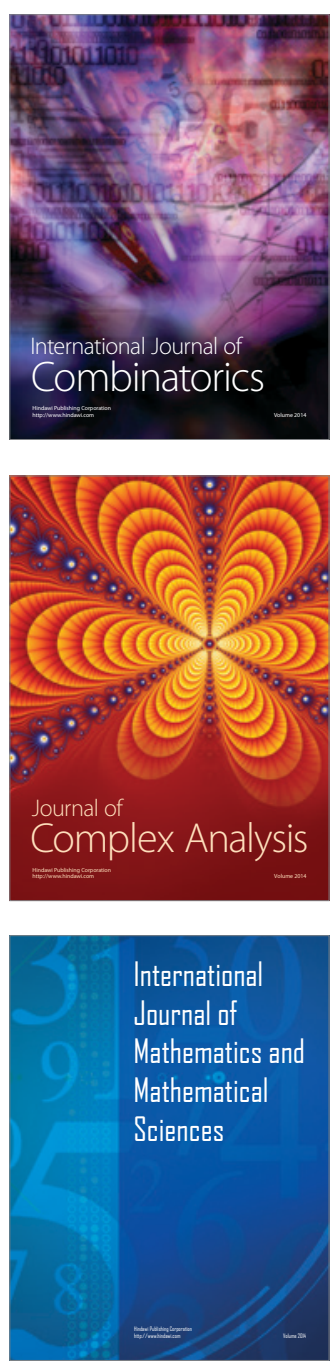
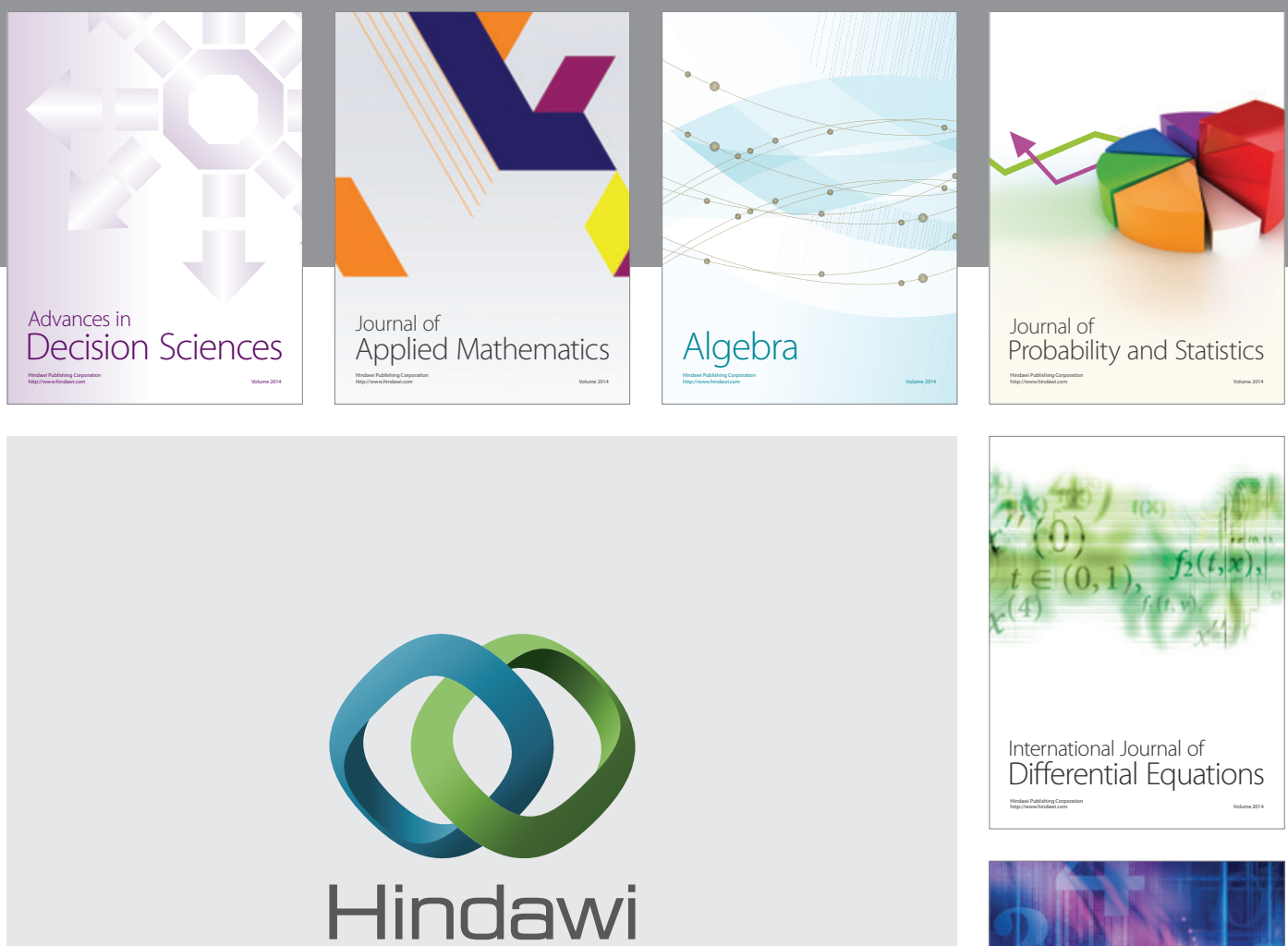

Submit your manuscripts at http://www.hindawi.com
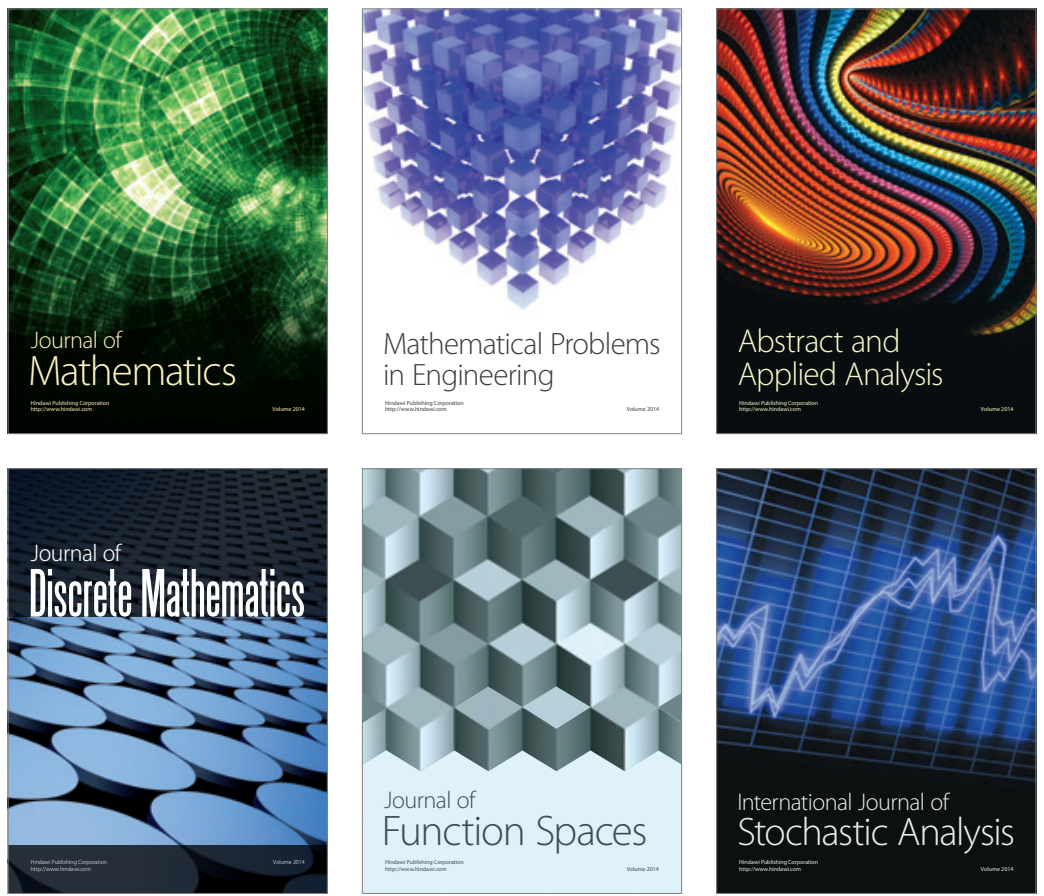

Journal of

Function Spaces

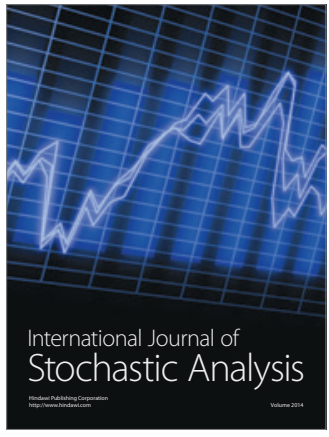

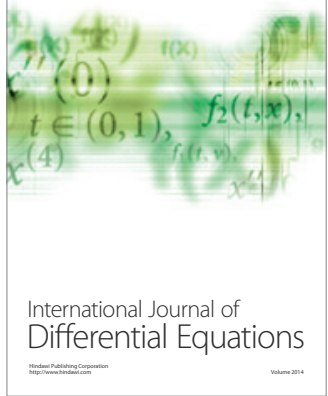
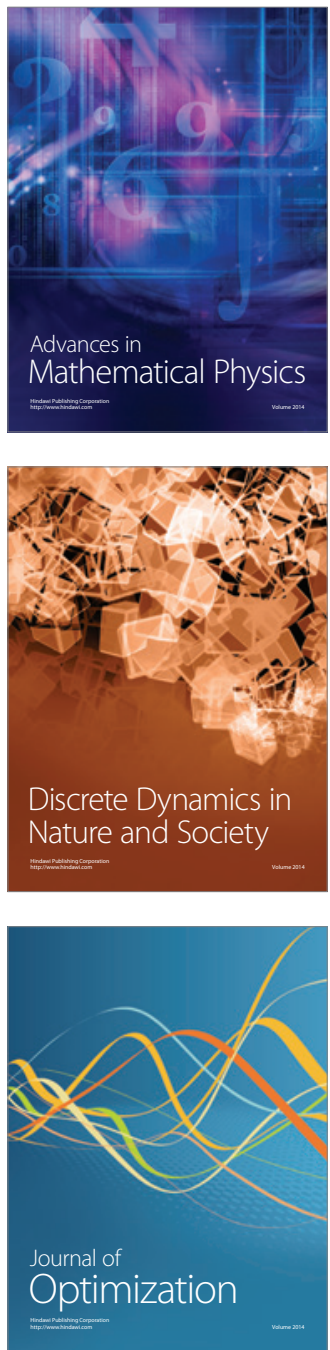